\title{
Non-Parametric Estimation of the Periodic Signal Parameters in the Frequency Domain
}

\author{
Dušan Agrež \\ University of Ljubljana, \\ Faculty of Electrical Engineering \\ Slovenia
}

\section{Introduction}

Parameters estimations of periodic signals $g(t)$, where frequency of the investigated component is the key parameter, play a fundamental role in a variety of applications: impedance measurement, power quality estimation, radar, A/D testing, etc. The problem of evaluating the spectral performance of a given periodic signal reduces to the parameter estimation of each spectral component (frequency, amplitude, and phase) in the presence of noise. Estimation methods can be classified as parametric (D'Antona \& Ferrero, 2006) and nonparametric (Agrež, 2002). Parametric methods are model-based and have very good selectivity and statistical efficiency, but require computationally intensive algorithms and very good 'model agreement' with a real multi-component signal. For this reason, such methods are unsuitable for many estimation problems. A better approach is to use nonparametric methods, which estimate the spectral parameters of interest by evaluating first the discrete Fourier transform (DFT) of the signal and then the parameters of the particular component. As we are dealing with periodic signals, the integral frequency transformation with the kernel $e^{-j 2 \pi f t}$ is, in principle, the best approximation to periodicity of the signal. Analysis of the frequency spectrum provides the opportunity to see systematic periodicities in the presence of the reduced random noise by integration. Many of these estimations are based on coherent sampling; that is, on the accurate synchronization of the signal and the sampling rate, and on the collection of a number of samples belonging to an integer number of the signal periods. However, the normal situation for signal parameter estimation is non-coherent, or quasi-coherent sampling, and in such a sampled signal there can also be spurious components. When failing to observe an integer number of periods of even a single tone, the tone energy is spread over the whole frequency axis, and the leakage from neighboring components can significantly bias estimations of the component parameters.

There are two fundamental principles that restrict estimations: the time-frequency uncertainty principle $\Delta T \Delta W \geq 1 / \pi$ (Gabor, 1946), and the principle of the limited changes of signals. The first, where $\Delta T$ and $\Delta W$ are the effective widths of lobes in the time and frequency domains (1), is a generalization of the Heisenberg uncertainty principle. 


$$
(\Delta T)^{2}=4[\underbrace{\frac{\int_{-\infty}^{\infty} t^{2} g^{2}(t) d t}{\int_{-\infty}^{\infty} g^{2}(t) d t}}-(\underbrace{\frac{\int_{-\infty}^{\infty} t g^{2}(t) d t}{\int_{-\infty}^{\infty} g^{2}(t) d t}})^{2}]
$$

The normalized moment of inertia about the center of gravity of the signal distribution.
The normalized first moment center of gravity of the signal distribution.

$$
(\Delta W)^{2}=4 \frac{\int_{-\infty}^{\infty} f^{2}|G(f)|^{2} d f}{\int_{-\infty}^{\infty}|G(f)|^{2} d f}
$$

In words, $\Delta T$ and $\Delta W$ cannot simultaneously be arbitrary small. In relation to measurements this is interpreted to imply that the uncertainty in the determination of a frequency, is of the order of magnitude of the reciprocal of the time taken to measure it.

The second principle, that of the limited changes of signals, limits the design of the signal shape. The more smoothly and slowly a function changes, the more rapidly its transformation changes and vice versa (Seibert, 1986). In practice this means that the spectrum of the signal should essentially vanish for frequencies greater than some frequency $f_{\max }$, and that the tails of the pulse in the time domain must die sufficiently rapidly that the tail of a large pulse will not seriously distort another smaller pulse at an adjacent time instant. The quantitative expression of this principle can be derived from Parseval's theorem and the differentiation properties of the Fourier transformation $\mathrm{d} g(t) / \mathrm{d} t \Leftrightarrow \mathrm{j} 2 \pi f G(f)$ :

$$
\int_{-\infty}^{\infty}\left|\frac{\mathrm{d}^{\mathrm{n}} g(t)}{\mathrm{d} t^{\mathrm{n}}}\right|^{2} \mathrm{~d} t=(2 \pi)^{2} \int_{-\infty}^{\infty} f^{2 n}|G(f)|^{2} \mathrm{~d} f
$$

Thus, if all the derivatives of the signal $g(t)$ through the $(n-1)^{\text {st }}$ are square-integrable, but the $n^{\text {th }}$ is not, we may in general conclude that its spectrum $G(f)$ vanishes faster than $|f|^{-n+1 / 2}$, but not faster than $|f|^{-n-1 / 2}$.

Both fundamental principles limit the accuracy of parameter estimations and depend upon the measurement time. Here this is taken to mean the relative time to measure a periodic signal, or the number of repetitions of the periodicity in it:

$$
\theta=\frac{T_{\text {meas }}}{T_{\text {period }}}=T_{\text {meas }} \cdot f=\frac{f}{\Delta f}
$$

where the measurement time determines the basic frequency resolution in the frequency domain $\Delta f=1 / T_{\text {meas }}$.

A finite time of measurement is a source of dynamic errors, which are shown as leakage parts of the measurement window spectrum, convolved on the spectrum of the measuredsampled signal (Fig. 1). The sampled analogue multi-frequency signal $g(t)$, by $f($ sampling $)=1 / \Delta t$, can be written as follows: 


$$
g(k \Delta t)_{N}=w(k) \cdot \sum_{m=0}^{M} A_{m} \sin \left(2 \pi f_{m} k \Delta t+\varphi_{m}\right), \quad k=0,1, \ldots, N-1
$$

where $f_{m}, A_{m}$, and $\varphi_{m}$ are frequency, amplitude, and phase of one component with index $m$ among $M+1$, respectively. Index $k$ is the current time index of the successive samples $\Delta t$ apart. Tones of the sampled signal do not generally coincide with the basic set of the periodic components of the DFT, which is the most well-known, non-parametric method for frequency decomposition of signals (Harris, 1978). Using $N$ samples of the signal (4), the DFT at the spectral line $i$ is given by:

$$
G(i)=-\frac{j}{2} \sum_{m=0}^{M} A_{m}\left[W\left(i-\theta_{m}\right) e^{j \varphi_{m}}-W\left(i+\theta_{m}\right) e^{-j \varphi_{m}}\right]
$$

where $W(*)$ is the spectrum of the used window $w(k)$, and $\theta_{m}$ is the signal component frequency divided by the frequency resolution $\Delta f=1 /(N \Delta t)$, and can be written in two parts:

$$
\theta_{m}=\frac{f_{m}}{\Delta f}=i_{m}+\delta_{m} \quad-0.5<\delta_{m} \leq 0.5
$$

where $i_{m}$ is an integer value and the displacement term $\delta_{m}$ is caused by the non-coherent sampling. The DFT coefficients surrounding one signal component are due to both the short-range leakage and the long-range leakage contributions from the second term of the investigated component, and from both terms of other components (7) (Fig. 1).

$$
\left|G\left(i_{m}\right)\right|=\frac{A_{m}}{2}\left|W\left(\delta_{m}\right) e^{j \varphi_{m}}-W\left(2 i_{m}+\delta_{m}\right) e^{-j \varphi_{m}}\right|+\sum_{k=0, k \neq m}^{M}\left|\Delta\left(i_{k}\right)\right|
$$

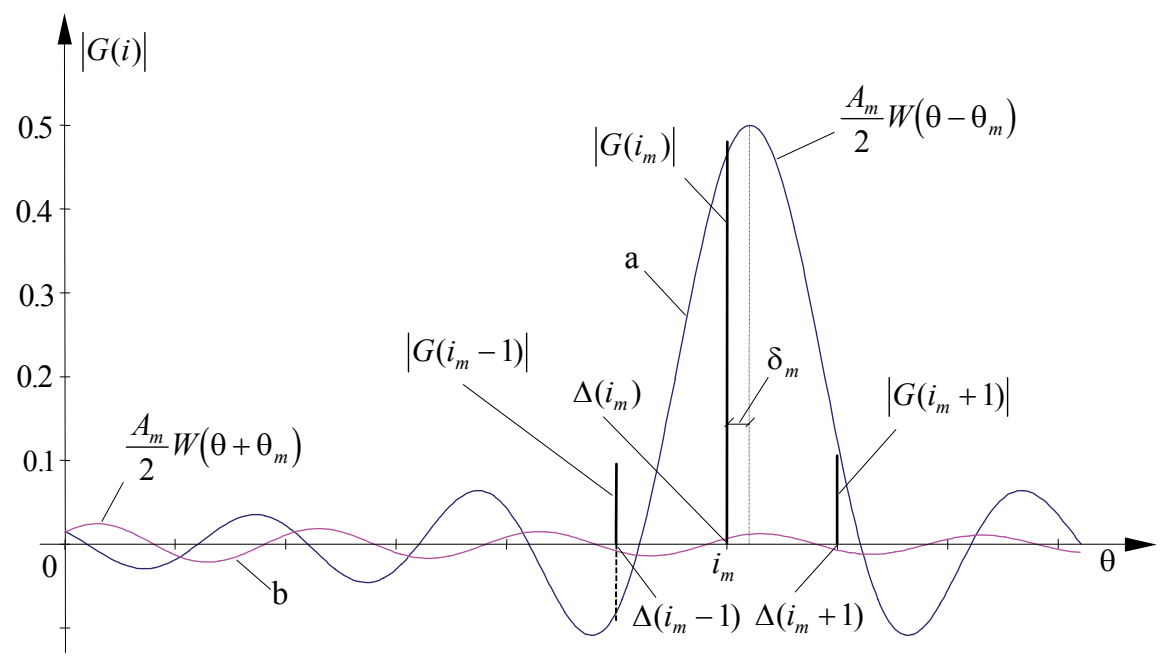

Fig. 1. The short-range leakage influences (a) and long-range leakage influences (b) on the amplitude DFT coefficients (rectangular window; $\theta_{m}=6.3$ ) 
The long-range leakage contributions can be reduced in several ways: by increasing the measurement time, by using windows with a faster reduction of the side lobes than with a rectangular window (like the Hann window, Rife-Vincent windows, Dolph-Chebychev windows, etc., Fig. 2), or by using the multi-point interpolated DFT algorithms and a window with known behavior of the spectrum (Agrež, 2002). For the sake of analytical simplicity, cosine-class windows are frequently used (Belega \& Dallet, 2009; Novotný \& Sedláček, 2010). The three basic classes of cosine windows were defined RV1, RV2, and RV3. For analyses, the first two classes are interesting.

Windows of the class RV1 (Fig. 2: curves $a, b$, and c) are designed for maximization of the window spectrum side-lobes fall-off $\theta^{-b}$, based on the number of the time domain window derivatives zeroes at the window ends (Novotný \& Sedláček, 2010):

$$
w(k)=\sum_{l=0}^{b-1}(-1)^{l} a_{l, 1} \cdot \cos \left(l \frac{2 \pi}{N} \cdot k\right), k=0, \ldots, N-1
$$

When the order $b$ is 1 (RV1-1), the coefficient $a_{0,1}$ is 1 and the equation (8) gives the rectangular shape. If $b$ is $2\left(R V 1-2: a_{0,1}=1 / 2, a_{1,1}=1 / 2\right)$ we get the Hann window. Higher values of $b$ (RV1-4: $\left.a_{0,1}=10 / 32, a_{1,1}=15 / 32, a_{2,1}=6 / 32, a_{3,1}=1 / 32\right)$ expand the window transform main-lobe and reduce the spectral leakage.

Windows of the class RV2 (Fig. 2: curves $\mathrm{d}$ and e) are designed for minimization of the window spectrum main-lobe width, for a given maximum level of the side-lobes relative magnitude $\left(A_{\text {side-lobe }} / A(0)\right)_{\max }=10^{-d}$, where $d$ is the exponent of the damping. They are the Taylor approximation to the Dolph-Chebychev windows and give good results when spectral components are very close (Andria et al., 1989).

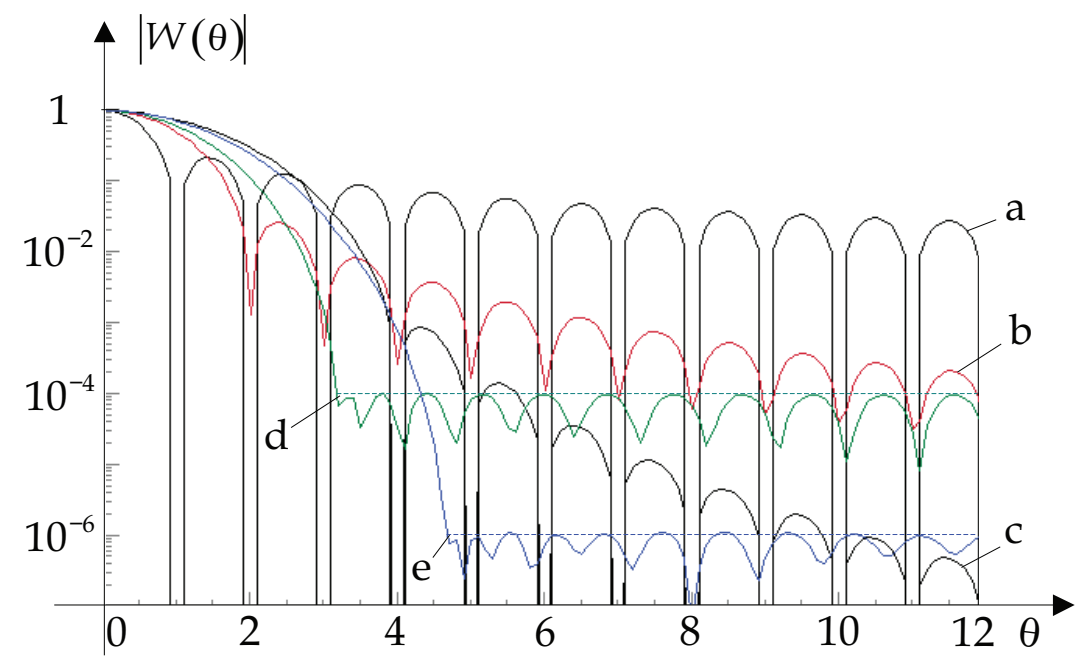

Fig. 2. Spectra shapes of the windows: $a$ - the rectangular window (RV1-1), b - the Hann window (RV1-2), c - the RV1-4 window, d - the RV2 window with $d=4$ (RV2-4), e - the RV2 window with $d=6$ (RV2-6) 


\section{Parameters estimations}

It is important how the used window works in the estimation. Especially in the first step if we have an iterative procedure of estimation. Estimation of the first step has the nonparametric nature since there is no information about the signal at the beginning.

\subsection{Frequency estimation}

Parameters of the measurement component can be non-parametric estimates by means of the interpolation. From the comparative study (Schoukens et al., 1992) it can be concluded that the key for estimating the three basic parameters is in determining the position of the measurement component $\delta_{m}=\theta_{m}-i_{m}$, between DFT coefficients $G\left(i_{m}\right)$ and $G\left(i_{m}+1\right)$, surrounding the component $m$ (Fig. 1.). Estimation can be done by multi-point estimations (Agrež, 2002) and using windows with known spectra, like the Hann window:

$$
\begin{gathered}
\left|W_{\mathrm{H}}(\theta)\right|_{N \gg 1}=\frac{|\sin (\pi \theta)|}{\left|2 \pi \theta\left(1-\theta^{2}\right)\right|} \text { in }\left|G\left(i_{m}\right)\right| \doteq \frac{A_{m}}{2}\left|W\left(\delta_{m}\right)\right| \\
\text { for 2-points estimation: }{ }_{2} \delta_{m} \doteq s \frac{2\left|G\left(i_{m}+s\right)\right|-\left|G\left(i_{m}\right)\right|}{\left|G\left(i_{m}\right)\right|+\left|G\left(i_{m}+s\right)\right|}
\end{gathered}
$$

where $s=\operatorname{sign}\left(\delta_{m}\right)$ is the sign of displacement and can be estimated by the difference of the phase DFT coefficients: $s=\operatorname{sign}\left(\left|\arg \left[G\left(i_{m}\right)\right]-\arg \left[G\left(i_{m}+1\right)\right]\right|-\pi / 2\right)$.

The three largest, local DFT coefficients can be used in the three-point interpolation and in this case, long-range leakage contributions can be considered. Portions $|\Delta(i)|(7)$ of the longrange leakage tails have the following properties: they decrease with increasing frequency and they change sign at successive coefficients $|G(i)|$, if they have a sine function in the kernel $\left(\sin \left(\pi\left(i+\delta_{m}\right)\right)=-\sin \left(\pi\left(i \pm 1+\delta_{m}\right)\right)\right)$. For example, the rectangular window, the Hann window, and Rife-Vincent Class I windows satisfy this condition. For $\theta_{m}$ that is large enough, the long-range leakage influence can be approximated to $\left|\Delta\left(i_{m}-1\right)\right| \approx\left|\Delta\left(i_{m}\right)\right| \approx\left|\Delta\left(i_{m}+1\right)\right|$, so that the ratio of coefficients can be expressed as:

$$
\begin{aligned}
{ }_{3} \alpha_{m} & =\frac{\left|G\left(i_{m}\right)\right|+\left|G\left(i_{m}-1\right)\right|}{\left|G\left(i_{m}\right)\right|+\left|G\left(i_{m}+1\right)\right|}= \\
& =\frac{\left|W\left(\delta_{m}\right)\right|+\left|\Delta\left(i_{m}\right)\right|+\left|W\left(1+\delta_{m}\right)\right|-\left|\Delta\left(i_{m}-1\right)\right|}{\left|W\left(\delta_{m}\right)\right|+\left|\Delta\left(i_{m}\right)\right|+\left|W\left(1-\delta_{m}\right)\right|-\left|\Delta\left(i_{m}+1\right)\right|} \approx \frac{\left|W\left(\delta_{m}\right)\right|+\left|W\left(1+\delta_{m}\right)\right|}{\left|W\left(\delta_{m}\right)\right|+\left|W\left(1-\delta_{m}\right)\right|}
\end{aligned}
$$

and the displacement term as:

$$
{ }_{3} \delta_{m} \cong 2 \frac{1-{ }_{3} \alpha_{m}}{1+{ }_{3} \alpha_{m}}=2 \frac{\left|G\left(i_{m}+1\right)\right|-\left|G\left(i_{m}-1\right)\right|}{\left|G\left(i_{m}-1\right)\right|+2\left|G\left(i_{m}\right)\right|+\left|G\left(i_{m}+1\right)\right|}
$$

The numerators and the denominators in (9) and (11) have the form where the amplitude DFT coefficients are added with suitable weights. The form of the denominator in (11) $\left|G_{\mathrm{H}}(i-1)\right|+2\left|G_{\mathrm{H}}(i)\right|+\left|G_{\mathrm{H}}(i+1)\right|$ is very characteristic, and looks like the form in the 
construction of the Hann window spectrum with the Dirac delta function $D(*)$, and the spectrum of the rectangular window (Harris, 1978):

$$
W_{\text {Hann. }}(\theta)=\left(\frac{1}{2} D(i)-\frac{1}{4} D(i-1)-\frac{1}{4} D(i+1)\right) \otimes W_{\text {rect. }}(\theta)
$$

but instead of the rectangular window, the Hann window can be used:

$$
W(\theta)=(D(i-1)+2 D(i)+D(i+1)) \otimes W_{\text {Hann. }}(\theta)
$$

From the point of view of leakage, the denominator is a sum of the weighted leakages. We can get the weights with a triple subtraction of the long-range leakage tails:

$$
\begin{aligned}
\left|\Delta\left(i_{m}-1, i_{m}\right)\right|=\left|\Delta\left(i_{m}-1\right)\right|-\left|\Delta\left(i_{m}\right)\right| ; \quad\left|\Delta\left(i_{m}, i_{m}+1\right)\right|=\left|\Delta\left(i_{m}\right)\right|-\left|\Delta\left(i_{m}+1\right)\right| & \begin{aligned}
\left|\Delta\left(i_{m}-1, i_{m}, i_{m}+1\right)\right| & =\left|\Delta\left(i_{m}-1, i_{m}\right)\right|-\left|\Delta\left(i_{m}, i_{m}+1\right)\right| \\
& =\left|\Delta\left(i_{m}-1\right)\right|-2\left|\Delta\left(i_{m}\right)\right|+\left|\Delta\left(i_{m}+1\right)\right|<<\left|\Delta\left(i_{m}\right)\right|
\end{aligned}
\end{aligned}
$$

The numerator is a subtraction of the sum of the first two, from the sum of the last two DFT coefficients $\left(\left|G\left(i_{m}\right)\right|+\left|G\left(i_{m}+1\right)\right|\right)-\left(\left|G\left(i_{m}-1\right)\right|+\left|G\left(i_{m}\right)\right|\right)=\left|G\left(i_{m}+1\right)\right|-\left|G\left(i_{m}-1\right)\right|$. In this case the long-range leakage tails are also reduced.

$$
\left(\left|\Delta\left(i_{m}\right)\right|-\left|\Delta\left(i_{m}+1\right)\right|\right)-\left(-\left|\Delta\left(i_{m}-1\right)\right|+\left|\Delta\left(i_{m}\right)\right|\right)=\left|\Delta\left(i_{m}-1\right)\right|-\left|\Delta\left(i_{m}+1\right)\right|
$$

It is appropriate to form multi-point interpolations on an odd number of coefficients, in order to have symmetry around the largest local coefficient $\left|G\left(i_{m}\right)\right|$. In a five-point interpolation with the Hann window, similar averages are used as in the three-point interpolation. The quotient is used to eliminate the amplitude influence of the investigated component.

$$
{ }_{5} \delta_{m}=3 \cdot \frac{2\left[\left|G\left(i_{m}+1\right)\right|-\left|G\left(i_{m}-1\right)\right|\right]+s \cdot\left[\left|G\left(i_{m}+2\right)\right|+\left|G\left(i_{m}-2\right)\right|\right]}{6\left|G\left(i_{m}\right)\right|+4\left[\left|G\left(i_{m}+1\right)\right|+\left|G\left(i_{m}-1\right)\right|\right]+s \cdot\left[\left|G\left(i_{m}+2\right)\right|-\left|G\left(i_{m}-2\right)\right|\right]}
$$

An estimation of the periodic parameter by the interpolation of the DFT gives the same effect as the reduction of spectrum tails. The meaning of the interpolation is the weighted summation of the amplitude coefficients, or better, symmetrical subtraction of the successive adjacent leakage parts of the window spectrum (14). The idea for long-range leakage reduction by summation of the adjacent weighted DFT coefficients, is at the core of the construction of the cosine class windows. Weights for forming the Hann window and the Rife-Vincent Class I windows from the rectangular window, are obtained by repeated convolution of the two-point weight pairs $(1,1)$, that is by repeated subtraction of the neighboring pairs of the spectrum leakage tails. Binomial weights $\left(\begin{array}{l}2 r \\ j\end{array}\right)(r=1,2, \ldots$ is a number of coefficients of one half; $j=0,1, \ldots, 2 r$ ) can be obtained from a Pascal triangle.

The displacement estimations with the multi-point $(\eta=2 r+1=3,5,7, \ldots)$ interpolations of the DFT using the Hann window can be written as: 


$$
\begin{gathered}
{ }_{2 r+1} \delta_{m} \cong(r+1) \cdot \frac{K_{n_{1}}\left[\left|G\left(i_{m}+1\right)\right|-\left|G\left(i_{m}-1\right)\right|\right]+\ldots+(-1)^{l} s K_{n_{l}}\left[\left|G\left(i_{m}+l\right)\right|+\left|G\left(i_{m}-l\right)\right|\right]}{K_{d_{1}}\left|G\left(i_{m}\right)\right|+K_{d_{2}}\left[\left|G\left(i_{m}+1\right)\right|+\left|G\left(i_{m}-1\right)\right|\right]+\ldots+(-1)^{l} s K_{d_{l}}\left[\left|G\left(i_{m}+l\right)\right|-\left|G\left(i_{m}-l\right)\right|\right]} \\
{ }_{\eta} \delta_{m} \cong\left(\frac{\eta+1}{2}\right) \cdot \frac{d_{\eta}}{n_{\eta}}
\end{gathered}
$$

where $l(l=1,2, \ldots \leq r)$ is the current index. The first term of the numerator in (17) is a difference of the side coefficients around the largest one, and the remaining terms are sums of the symmetrical pairs of coefficients $\left(\left|G\left(i_{m} \pm l\right)\right|\right)$. All terms [*] are weighted by $K_{n_{l}}=\left(\begin{array}{l}2(r-1) \\ (r-l)\end{array}\right)-\left(\begin{array}{c}2(r-1) \\ (r-l-2)\end{array}\right)$. The signs of weights alter successively. The largest coefficient in the denominator is weighted by $K_{d_{1}}=\left(\begin{array}{c}2 r \\ r\end{array}\right)$, the sum of the first side coefficients by $K_{d_{2}}=\left(\begin{array}{c}2 r \\ (r-1)\end{array}\right)$ and, the differences of the symmetrically located coefficients are weighted by $K_{d_{l+1}}=\left(\begin{array}{c}2 r \\ (r-l)\end{array}\right)$. The signs of weights also alter successively $(-1)^{l}$.

\subsubsection{Reduction of systematic error}

The results of the simulations, where the relative frequency was changed, show that the systematic contribution of the error - the estimation bias - decreases (Fig. 3.), while the influence of noise on estimations $\left(\sigma_{\theta_{m}}\right)$ increases with the number of interpolation points (Fig. 4.).

The errors of the frequency estimations $E(\theta)=(i+\delta)-\theta^{*} \quad\left(\theta^{*}\right.$ is the true value of the relative frequency) for one sine component in the signal have been checked with a double scan varying both frequency and phase $\left(A_{m}=1, N=1024,1 \leq \theta \leq 6, \Delta \theta=0.001\right.$ and $-\pi / 2 \leq \varphi \leq \pi / 2, \Delta \varphi=\pi / 180$ ). The absolute maximum values of the errors (from 181 iterations) at the given relative frequency were compared for the multi-point DFT interpolations using the Hann window (Fig. 3).

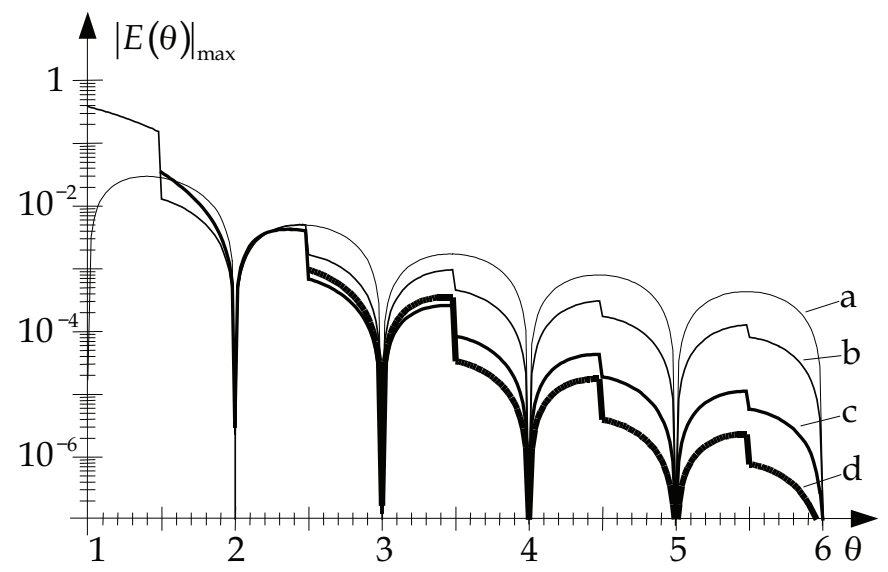

Fig. 3. Maximal errors of frequency estimation with the multi-point interpolations of the DFT for the Hann window (a: two-point interpolation; b: three-point interpolation; c: fivepoint interpolation; $\mathrm{d}$ : seven-point interpolation) 


\subsubsection{Uncertainty of the frequency estimations}

Distributions of errors $\left(E=|G(i)|_{\text {noise }}-|G(i)|_{\text {noiseless }}\right)$ of the largest amplitude DFT coefficients have very similar (Gaussian) shapes with almost equal standard deviations $\sigma_{|\mathrm{DFT}|}\left(i_{m}\right) \approx \sigma_{|\mathrm{DFT}|}\left(i_{m} \pm 1,2,.\right)=\sigma_{|\mathrm{DFT}|}$, if the time domain noise with standard deviation $\sigma_{t}$ and a mean value $u(t) \rightarrow 0$, is statistically independent of the signal and sampling process (18). In other words, if in the first approximation only the quantization noise is considered, the sampling frequency should be suitably larger than the frequency of the highest frequency component $\left(f_{M}\right) f_{S} / f_{M} \gg 2$ and the effective number of bits $(E N O B=b)$ of the A/D conversion suitably high enough $b \geq 3$, or $S N R \geq 20 \mathrm{~dB}$ (Widrow, Kollar. 2008). The signal to noise ratio in the time domain is defined as $S N R=A_{m}^{2} /\left(2 \sigma_{t}^{2}\right)(S N R / \mathrm{dB}=10 \log S N R)$, or expressed with an effective number of bits $S N R=3 / 2 \cdot\left(2^{b}\right)^{2}$, where the rectangular distribution of the quantization errors is taken into account $\sigma_{t}=A_{m} /\left(2^{b}\right) \cdot 1 / \sqrt{3}$.

$$
\sigma_{|\mathrm{DFT}|}=\sigma_{\mathrm{t}} \frac{1}{N \sqrt{2}} \sqrt{\sum_{k=0}^{N-1} w^{2}(k)}
$$

The "absolute" form of the standard deviation (18) is usually related to the values of the DFT coefficients of interest. In coherent sampling the largest local amplitude DFT coefficient is equal to $\left|G\left(i_{m}\right)\right|=A_{m} / 2 \cdot \sum w(k) / N$, where $\sum w(k) / N \leq 1$ represents the normalized peak signal gain of the window $w(k)$ (Solomon 1992). The relative form of the standard deviation can be written as:

$$
\sigma_{|\mathrm{DFT}|}^{*}=\frac{\sigma_{|\mathrm{DFT}|}}{\left|G\left(i_{m}\right)\right|}=\frac{\sigma_{x}}{A_{m}} \sqrt{2} \frac{\sqrt{\sum_{k=0}^{N-1} w^{2}(k)}}{\sum_{k=0}^{N-1} w(k)}=\frac{\sigma_{x}}{A_{m}} \frac{\sqrt{2}}{\sqrt{N}} \sqrt{E N B W}
$$

The root of the equivalent noise bandwidth ENBW (Harris, 1978) is a factor determining the size of the standard deviation when using different windows: $E N B W>E N B W_{\text {rect. }}=1$.

Distortions of the DFT coefficients and their number in an interpolation have a significant influence on the uncertainty of the displacement estimation. The standard deviation of $\delta_{m}$, as a dependent quantity, can generally be expressed as (Joint Committee for Guides in Metrology [JCGM], 2008):

$$
\sigma_{\delta_{m}}^{2}=\sum_{p=i_{m}-r}^{p=i_{m}+r}\left(c_{\delta}(p) \cdot \sigma_{|G(p)|}\right)^{2}+2 \sum_{p=i_{m}-r}^{p=i_{m}+r-1} \sum_{v=p+1}^{v=i_{m}+r}\left(r_{C}(|G(p)|,|G(v)|) \cdot c_{\delta}(p) \cdot c_{\delta}(v) \cdot \sigma_{|G(p)|} \cdot \sigma_{|G(v)|}\right)
$$

where $c_{\delta}(p)=\partial \delta_{m} / \partial|G(p)|\left(p=i_{m}-r, \ldots, i_{m}+r\right)$ is the sensitivity coefficient associated with the amplitude coefficient $|G(p)|$, and $r_{C}(|G(p)|,|G(v)|) \quad(p \neq v)$ is the correlation coefficient. In the case of the Hann window, two successive amplitude coefficients have the correlation factor $r_{\mathrm{C}}(|G(p)|,|G(p+1)|)=2 / 3$, and amplitude coefficients with a current index of two apart, have $r_{C}(|G(p)|,|G(p+2)|)=1 / 6$. Other correlation coefficients are zero. 
As the standard deviations of the amplitude coefficients are almost equal $\sigma_{|G(p)|} \cong \sigma_{|G(v)|}=\sigma_{|\mathrm{DFT}|}$, it is possible to formulate the expression for the standard deviation of displacement. For the three-point interpolation using the Hann window (11), it can be expressed as:

$$
\begin{aligned}
{ }_{3} \sigma_{\delta_{m}}^{2}=\sigma_{|\mathrm{DFT}|}^{2}( & \left(c_{\delta}^{2}\left(i_{m-1}\right)+c_{\delta}^{2}\left(i_{m}\right)+c_{\delta}^{2}\left(i_{m+1}\right)\right) \\
+ & \frac{4}{3}\left(c_{\delta}\left(i_{m}-1\right) \cdot c_{\delta}\left(i_{m}\right)+c_{\delta}\left(i_{m}\right) \cdot c_{\delta}\left(i_{m}+1\right)\right) \\
& \left.+\frac{2}{6}\left(c_{\delta}\left(i_{m}-1\right) \cdot c_{\delta}\left(i_{m}+1\right)\right)\right)
\end{aligned}
$$

The same mathematical procedure can be used for other higher multi-point interpolations (17).

$$
\left({ }_{\eta} \sigma_{\delta_{m}} / \sigma_{|\mathrm{DFT}|}\right)^{2}=\sum_{p=i_{m}-r}^{p=i_{m}+r} c_{\delta}^{2}(p)+\frac{4}{3} \cdot \sum_{p=i_{m}-r}^{p=i_{m}+r-1} c_{\delta}(p) \cdot c_{\delta}(p+1)+\frac{1}{3} \cdot \sum_{p=i_{m}-r}^{p=i_{m}+r-2} c_{\delta}(p) \cdot c_{\delta}(p+2)
$$

Sensitivity coefficients $c(p)$ have forms such as $c(p)=\left(\left(d_{\eta}\right)^{\prime} \cdot n_{\eta}-\left(n_{\eta}\right)^{\prime} \cdot d_{\eta}\right) / n_{\eta}^{2}$, where $d_{\eta}$, $n_{\eta}$ are the denominator and the numerator of fraction (17), respectively. Both are sums of the weighted amplitude DFT coefficients, which change with relative displacement - the short-range leakage influences. For this reason, the standard deviations of displacements change their values periodically $-0.5<\delta_{m} \leq 0.5$ (Fig. 4).

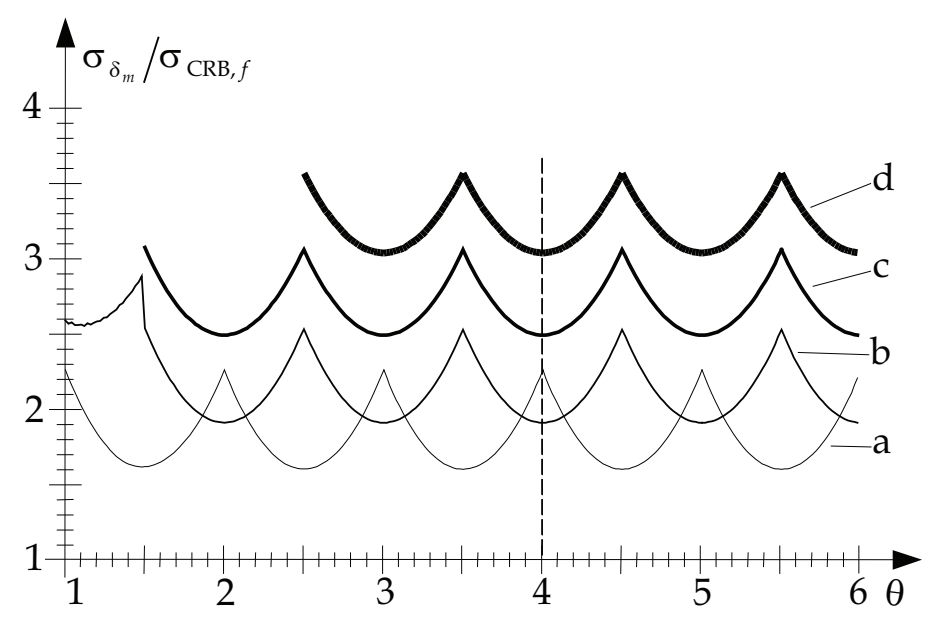

Fig. 4. Standard deviations of the displacement estimation related to the CRB standard deviation for the frequency estimation (a: two-point interpolation; b: three-point interpolation; c: five-point interpolation; d: seven-point interpolation)

The standard deviation of the displacement estimation is related to the absolute form of the standard deviation of the amplitude DFT coefficient with a suitable factor 
$\sigma_{\delta_{m}}=\sigma_{|\mathrm{DFT}|}{ }_{\eta} R_{\delta}$ according to (22). If one wants to compare it with the unbiased CramérRao lower bound (CRB) for the estimation of the frequency (Petri, 2002) the relationship has to be reexpressed:

$$
\sigma_{\delta_{m}}=\frac{\sigma_{|\mathrm{DFT}|}}{\left|G\left(i_{m}\right)\right|} \cdot\left(\left|G\left(i_{m}\right)\right| \cdot{ }_{\eta} R_{\delta}\right)=\frac{1}{\sqrt{S N R}} \frac{\sqrt{E N B W}}{\sqrt{N}} \cdot\left(\left|G\left(i_{m}\right)\right| \cdot{ }_{\eta} R_{\delta}\right) \geq \frac{1}{\sqrt{S N R}} \frac{1}{\sqrt{N}} \frac{\sqrt{3}}{\pi}=\sigma_{\mathrm{CRB}, f}
$$

This form is larger than $\sigma_{\mathrm{CRB}, f}$ for the frequency estimation taking into account all measurement information (Fig. 4).

Errors in relative frequency estimations with different numbers of interpolation points have normal distributions. The standard deviation of the three-point frequency estimation, which has the lowest standard deviation in the vicinity of the integer values of the relative frequency, is about 2.2 times higher than $\sigma_{\mathrm{CRB}, f}$ (Fig. 4: curve b). The lowest value $\left({ }_{3} \sigma_{\delta_{m}} / \sigma_{\mathrm{CRB}, f} \approx 1.9\right)$ is attained at $\delta_{m} \approx 0$, and the highest ratio $\left({ }_{3} \sigma_{\delta_{m}} / \sigma_{\mathrm{CRB}, f} \approx 2.55\right)$ is at the worst cases of the non-coherent sampling $\left(\delta_{m} \approx 0.5\right)$. A two-point interpolation is worse around $\delta_{m} \approx 0 \quad\left({ }_{2} \sigma_{\delta_{m}} / \sigma_{\mathrm{CRB}, f} \approx 2.26\right)$, but it is superior in the interval $0.15 \leq\left|\delta_{m}\right| \leq 0.5$ $\left({ }_{2} \sigma_{\delta_{m}} / \sigma_{\mathrm{CRB}, f} \geq 1.59\right)$.

\subsubsection{A trade-off between bias and uncertainty}

If we reduce the leakage tails, or systematic errors by the interpolation, we apparently widen the estimation main-lobe $\Delta W_{\text {est }}(E N B W>1)$, and the noise in the estimation increases in comparison to the CRB. For example, the noise of the cosine windows increases $\operatorname{ENBW}\left(\cos ^{2}(X)\right) / \operatorname{ENBW}\left(\cos ^{4}(X)\right) \approx 1.5 / 1.94 \approx 1 / 1.29$, while the side-lobes levels decrease $S L$ fall $\left(\cos ^{2}(X)\right) / S L$ fall $\left(\cos ^{4}(X)\right) \approx-18 \mathrm{~dB} /-30 \mathrm{~dB}$ (Harris, 1978). At the same time, the systematic errors $\left(\left|E_{\theta}\right|_{\max }\right)$ decrease with increasing numbers of points. Increasing the number of the used DFT coefficients is reasonable until the systematic error drops under the noise error. After this point, by increasing the relative frequency $\theta_{m}$, or with spacing between the two frequency components $\left(\propto 2 \cdot \theta_{m}\right)$, it is logical to decrease the number of interpolation points.

The criterion for selecting one of the algorithms could be the minimum common uncertainty of the estimation considering both contributions:

$$
\sigma_{\Sigma}=\sqrt{\left(\frac{\left|E\left(\theta_{m}\right)\right|_{\max }}{\sqrt{2}}\right)^{2}+\sigma_{\theta_{m}}^{2}} \rightarrow \min
$$

The effective value of the systematic contribution is obtained by dividing the maximal error by the square root of two, since systematic errors are phase dependent with a sine like shape.

The borders of relative frequency where one interpolation can pass over another depend upon the number $b$ of bits of the A/D converter. With a 10-bit A/D converter $(S N R \approx 62 \mathrm{~dB})$, which is frequently used in industrial environments, it is convenient to use the three-point DFT interpolation with the Hann window, in the interval $1<\theta<2.2$ and 
from $6.7<\theta$ onward (Fig. 5). Between values 2.2 and 6.7 of the relative frequency, it is better to use the five-point interpolation (or even the seven-point interpolation in intervals $[3.5,3.8]$ and $[4.3,4.5])$.

Multi-point interpolations present worse results: at lower $\theta$ owing to systematic error ("window width"), and at higher values of $\theta$ owing to the larger noise sensibility. The solid line at the top of Fig. 5 shows where different multi-point interpolations can be used to achieve the best results of the one-component frequency estimation.

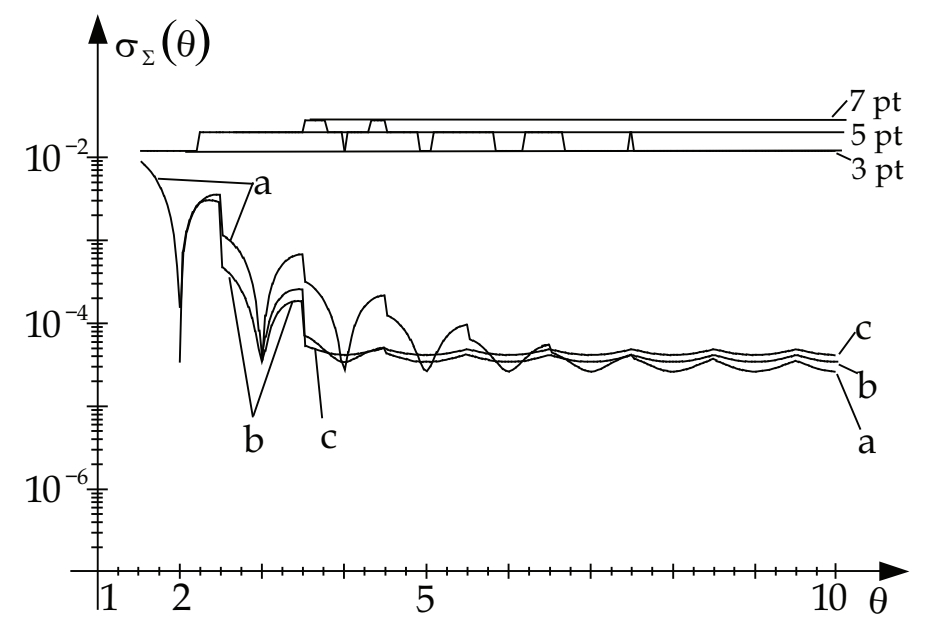

Fig. 5. The use of multi-point DFT interpolations for a 10-bit A/D converter (a: three-point interpolationb: five-point interpolationc: seven-point interpolation)

\subsection{Amplitude estimation}

From the behavior of the systematic error of the frequency estimation (Fig. 3), it can be concluded that it is better to use the Hann, or some higher order cosine window for the estimation, if the window spectrum is analytically known. When the displacement $\delta_{m}$ for the specific component is determined, it is easy to get the amplitude using the Hann window (9a) and neglecting the long-range contribution $\left|\Delta\left(i_{m}\right)\right|$ in (7):

$$
A_{m}=2\left|\frac{2 \pi \delta_{m}\left(1-\delta_{m}^{2}\right)}{\sin \left(\pi \delta_{m}\right)}\right| G\left(i_{m}\right) \mid
$$

As in the case of the frequency estimation, with the summation of the DFT coefficients, we subtract the long-range leakage tails and reduce their influences. We get the weights for the three-point summation with the triple subtraction of the long-range leakage tails (14).

$$
\left|\Delta\left(i_{m}-1\right)\right|-2\left|\Delta\left(i_{m}\right)\right|+\left|\Delta\left(i_{m}+1\right)\right|<<\left|\Delta\left(i_{m}\right)\right|
$$

In this manner, we can get the amplitude of the signal by summing the largest three local DFT coefficients around the signal component following the result of (26): 


$$
\begin{gathered}
{\left[\left|G\left(i_{m}-1\right)\right|+2\left|G\left(i_{m}\right)\right|+\left|G\left(i_{m}+1\right)\right|\right]=\frac{A_{m}}{2}\left[\left|W\left(1+\delta_{m}\right)\right| \pm\left|\Delta\left(i_{m}-1\right)\right|+\right.} \\
\left.+2\left|W\left(\delta_{m}\right)\right| \mp 2\left|\Delta\left(i_{m}\right)\right|+\left|W\left(1-\delta_{m}\right)\right| \pm\left|\Delta\left(i_{m}+1\right)\right|\right] \\
A_{m} \cong 2 \cdot \frac{\left[\left|G\left(i_{m}-1\right)\right|+2\left|G\left(i_{m}\right)\right|+\left|G\left(i_{m}+1\right)\right|\right]}{\left|W\left(1+\delta_{m}\right)\right|+2\left|W\left(\delta_{m}\right)\right|+\left|W\left(1-\delta_{m}\right)\right|}
\end{gathered}
$$

Using the Hann window:

$$
\begin{gathered}
\left|W_{\mathrm{H}}\left(\delta_{m}\right)\right| \cong \frac{\sin \left(\pi \delta_{m}\right)}{2 \pi \delta_{m}\left(1-\delta_{m}^{2}\right)} \text { and }\left|W_{\mathrm{H}}\left(1+s \delta_{m}\right)\right| \cong \frac{\sin \left(\pi \delta_{m}\right)}{2 \pi \delta_{m}\left(1+s \delta_{m}\right)\left(2+s \delta_{m}\right)} \\
\left|W\left(1+\delta_{m}\right)\right|+2\left|W\left(\delta_{m}\right)\right|+\left|W\left(1-\delta_{m}\right)\right|=\frac{\sin \left(\pi \delta_{m}\right)}{2 \pi \delta_{m}} \frac{12}{\left(1-\delta_{m}^{2}\right)\left(4-\delta_{m}^{2}\right)}
\end{gathered}
$$

the amplitude estimation with the three-point interpolation $\left({ }_{3} A_{m \mathrm{H}}\right)$ can be expressed as follows:

$$
{ }_{3} A_{m \mathrm{H}} \cong \frac{\pi \delta_{m}}{\sin \left(\pi \delta_{m}\right)} \frac{\left(1-\delta_{m}^{2}\right)\left(4-\delta_{m}^{2}\right)}{3} \cdot\left[\left|G\left(i_{m}-1\right)\right|+2\left|G\left(i_{m}\right)\right|+\left|G\left(i_{m}+1\right)\right|\right]
$$

We can use the same procedure for the five-point interpolation with ten subtractions of the tails. In the first step of the procedure we do four subtractions of the adjacent tails $\left|\Delta\left(i_{m}-2, i_{m}-1\right)\right|, \ldots,\left|\Delta\left(i_{m}+1, i_{m}+2\right)\right|$ as in (14), or summations of the DFT coefficients, then three subtractions of the obtained and reduced tails as in (26), and so on. After rearrangement the amplitude can be expressed with the weighted five largest coefficients:

$$
\begin{aligned}
{ }_{5} A_{m \mathrm{H}} & =\frac{\pi \delta_{m}}{\sin \left(\pi \delta_{m}\right)} \frac{\left(1-\delta_{m}^{2}\right)\left(4-\delta_{m}^{2}\right)\left(9-\delta_{m}^{2}\right)}{90} . \\
& \cdot\left[6\left|G\left(i_{m}\right)\right|+4\left(\left|G\left(i_{m}+1\right)\right|+\left|G\left(i_{m}-1\right)\right|\right)+|| G\left(i_{m}+2\right)|-| G\left(i_{m}-2\right)||\right]
\end{aligned}
$$

In the last term of the equation (31), the absolute value of the difference is used, since one of

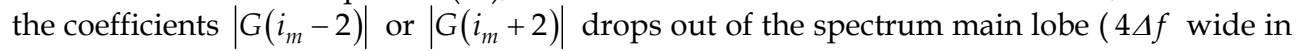
the Hann case) and gets a negative sign.

The same procedure can be used for the seven-point interpolation:

$$
\begin{aligned}
{ }_{7} A_{m \mathrm{H}}= & \frac{\pi \delta_{m}}{\sin \left(\pi \delta_{m}\right)} \frac{\left(1-\delta_{m}^{2}\right)\left(4-\delta_{m}^{2}\right)\left(9-\delta_{m}^{2}\right)\left(16-\delta_{m}^{2}\right)}{5040} . \\
& \cdot\left[20 \cdot\left|G\left(i_{m}\right)\right|+15 \cdot\left(\left|G\left(i_{m}+1\right)\right|+\left|G\left(i_{m}-1\right)\right|\right)+\right. \\
& \left.+6 \cdot|| G\left(i_{m}+2\right)|-| G\left(i_{m}-2\right)||-|| G\left(i_{m}+3\right)|-| G\left(i_{m}-3\right)||\right]
\end{aligned}
$$


The relative error $e(A)=\left(A / A^{*}-1\right)\left(A^{*}=1\right.$ is the true value of the amplitude) drops with increasing relative frequency and with the number of the interpolation points (Fig. 6: ${ }_{0} e-$ the amplitude is estimated only with the largest coefficient, ${ }_{1} e$ - estimation with (25), ${ }_{3} e-$ estimation with (30), etc; The same testing conditions as for Fig. 3). Comparing figures 6 and 7 shows the importance of the frequency estimation accuracy. If we know the value of the

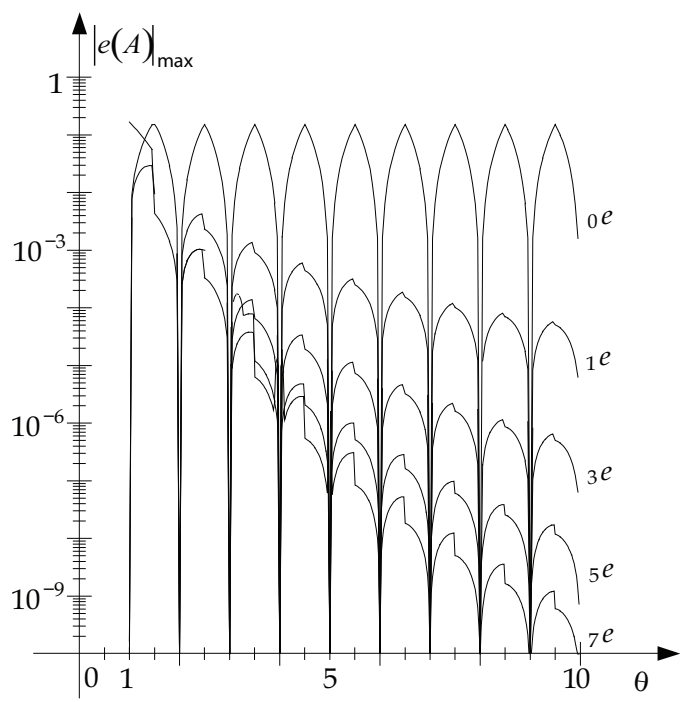

Fig. 6. Maximal relative values of errors of the amplitude estimation with the multi-point DFT interpolations with the Hann window ( $\theta$ is known)

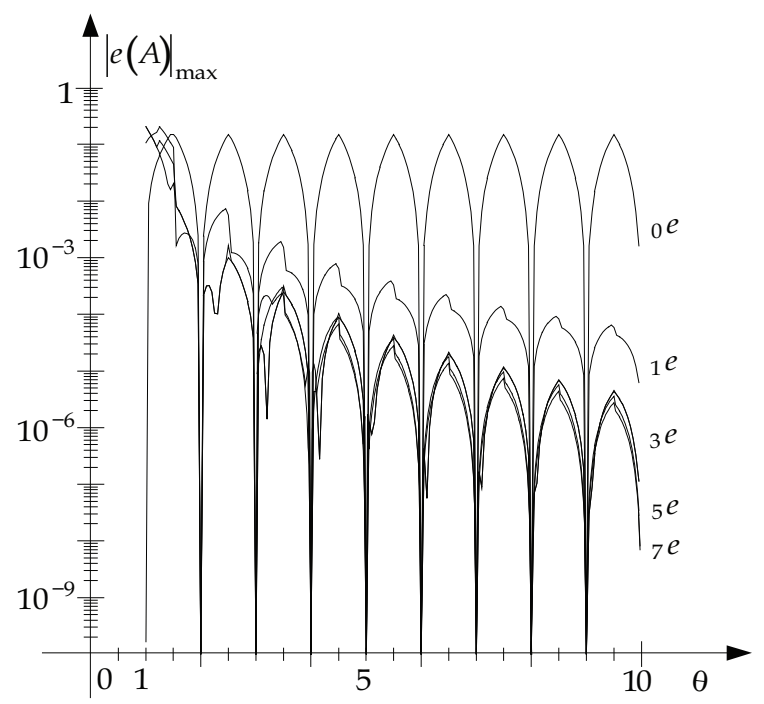

Fig. 7. Maximal relative values of errors of the amplitude estimation with the multi-point DFT interpolations with the Hann window ( $\theta$ is obtained with the three-point int. (11)) 
frequency on the three-point interpolation accuracy level, then the amplitude estimation is reasonable with the three-point interpolation. The accuracy of the amplitude estimation can be improved, if the frequency is better estimated (e.g., by the multipoint interpolations).

\subsubsection{Influence of noise on the amplitude estimation}

Uncertainty of the component amplitude estimation mainly depends on the uncertainties of the amplitude DFT coefficients. Equation (19) is valid for all amplitude coefficients of the DFT that are large enough and sufficiently (half of the main lobe width) moved away from the margins of the spectral field $(\theta=0, N / 2)$.

The price for the effective leakage reduction is in the increase of the estimation uncertainties, related to the unbiased CRB fixed by the signal-to-noise-ratio for a particular component. In Fig. 8, there are standard uncertainties of the amplitude estimation related to the CRB (33) (Petri, 2002) for the three-point estimation.

$$
\sigma_{A_{m}} \geq \frac{1}{\sqrt{S N R}} \frac{1}{\sqrt{N}}=\sigma_{\mathrm{CRB}, A}
$$

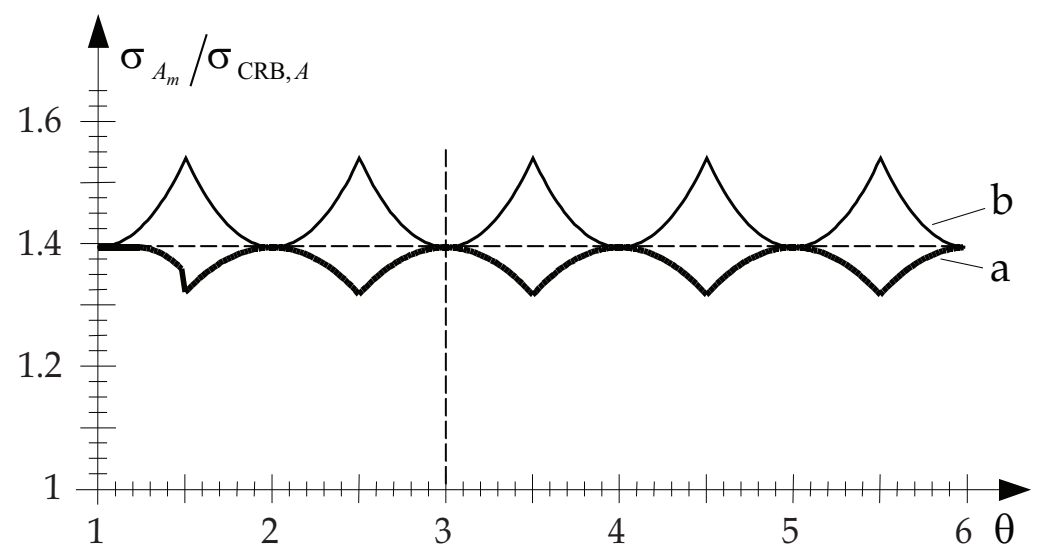

Fig. 8. Standard uncertainty of the amplitude three-point estimation using the Hann window related to the CRB (33) (a: $\theta$ is estimated, b: $\theta$ is known)

The distortions of the DFT coefficients and the number of points in the interpolation, have significant influence on the uncertainty of displacement $\delta_{m}$, and amplitude $A_{m}$, succesively. As with the frequency estimation, the systematic errors decrease when increasing the number of points. Increasing the number of DFT coefficients used in the interpolation is reasonable, until the systematic error drops under the noise error (Fig. 9). After this point, by increasing the relative frequency $\theta_{m}$ (the number of periods of the measured signal in the measurement interval), or increasing spacing between two frequency components $\left(\propto 2 \cdot \theta_{m}\right)$, the number of interpolation points can be decreased. A smaller number of the DFT coefficients in the calculation produces lower noise distortion, which becomes dominant in the final result. 


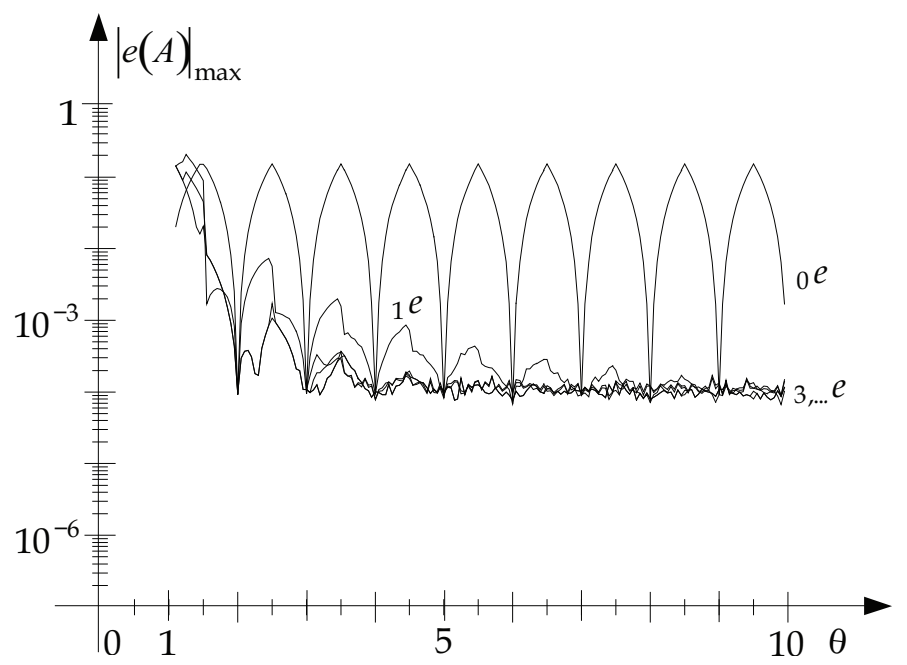

(a)

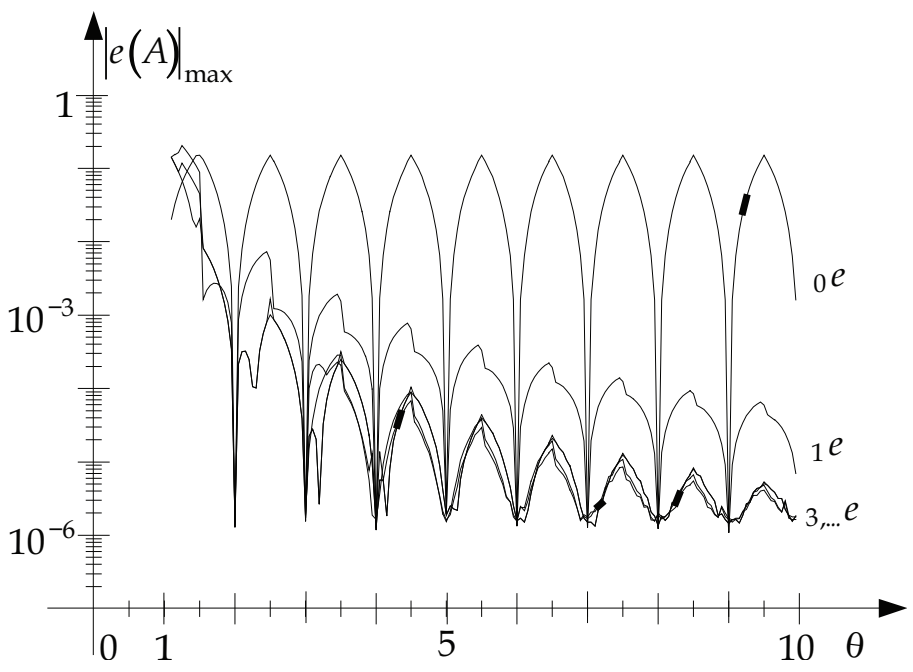

(b)

Fig. 9. The influence of the quantization noise on the amplitude estimation with 10-bit A/D converter (a) and 16-bit A/D converter (b)

\subsection{Phase estimation}

The second parameter of the signal component, besides the amplitude of the frequency main lobe, is the phase, i.e. the time position of the signal structure. As with previous estimations, the function $W(\theta)$ has to be analytically known. For the rectangular window with a large number of points $N>>1$, the following equation is valid, where the Dirichlet kernel is used (Harris, 1978): 


$$
W_{\text {rect. }}(\theta)=\frac{\sin (\pi \theta)}{N \sin (\pi \theta / N)} \cdot \mathrm{e}^{-\mathrm{j} \pi\left(\frac{N-1}{N}\right) \theta}
$$

The largest DFT coefficient, which is mostly composed of the short-range leakage contribution of the investigated component $m$, can be deduced from (5), and (34) using $a=\pi(N-1) / N$ and $-\mathrm{j}=\mathrm{e}^{-\mathrm{j} \pi / 2}$ :

$$
G\left(i_{m}\right)=\frac{A_{m}}{2}\left(\frac{\sin \left(\pi\left(i_{m}-\theta_{m}\right)\right)}{N \sin \left(\pi\left(i_{m}-\theta_{m}\right) / N\right)} \mathrm{e}^{\mathrm{j}\left(a\left(\theta_{m}-i_{m}\right)+\varphi_{m}-\frac{\pi}{2}\right)}-\frac{\sin \left(\pi\left(i_{m}+\theta_{m}\right)\right)}{N \sin \left(\pi\left(i_{m}+\theta_{m}\right) / N\right)} \mathrm{e}^{-\mathrm{j}\left(a\left(\theta_{m}+i_{m}\right)+\varphi_{m}+\frac{\pi}{2}\right)}\right)
$$

The component phase $\varphi_{m}$ is referred to as the start point of the window (not the middle point as is the case with the frequency and amplitude).

As $N$ is usually large $N>>1$, and considering (6), equation (35) can be rewritten:

$$
G\left(i_{m}\right)=\frac{A_{m}}{2}\left(\frac{\sin \left(\pi \delta_{m}\right)}{\pi \delta_{m}} \mathrm{e}^{\mathrm{j}\left(a\left(\delta_{m}\right)+\varphi_{m}-\frac{\pi}{2}\right)}-\frac{\sin \left(\pi \delta_{m}\right)}{\pi\left(2 i_{m}+\delta_{m}\right)} \mathrm{e}^{-\mathrm{j}\left(a\left(2 i_{m}+\delta_{m}\right)+\varphi_{m}+\frac{\pi}{2}\right)}\right)
$$

Both, amplitude and phase have additional disturbing components from the second part in (36):

$$
G\left(i_{m}\right)=\left|G\left(i_{m}\right)\right|^{\mathrm{j}\left(a\left(\delta_{m}\right)+\varphi_{m}-\frac{\pi}{2}\right)} \pm \Delta\left(i_{m}\right), \quad \arg \left(G\left(i_{m}\right)\right)=\varphi_{m}+a \delta_{m}-\frac{\pi}{2} \pm \Delta \varphi\left(i_{m}\right)
$$

If the displacement term is positive $0.5>\delta_{m} \geq 0$, then the second largest DFT coefficient is $G\left(i_{m}+1\right)$, and if the displacement term is negative $0>\delta_{m} \geq-0.5$, then the second largest DFT coefficient is $G\left(i_{m}-1\right)$ (Fig. 10). The largest side coefficient may commonly be expressed as:

$$
\begin{gathered}
G\left(i_{m}+s\right)=\frac{A_{m}}{2}\left(\frac{\sin \left(\pi\left(s-\delta_{m}\right)\right)}{\pi\left(s-\delta_{m}\right)} \mathrm{e}^{\mathrm{j}\left(a\left(\delta_{m}-s\right)+\varphi_{m}-\frac{\pi}{2}\right)}-\frac{\sin \left(\pi\left(2 i_{m}+s+\delta_{m}\right)\right)}{\pi\left(2 i_{m}+s+\delta_{m}\right)} \mathrm{e}^{-\mathrm{j}\left(a\left(\delta_{m}-s\right)+\varphi_{m}-\frac{\pi}{2}\right)}\right) \\
G\left(i_{m}+s\right)=\left|G\left(i_{m}+s\right)\right| \mathrm{e}^{\mathrm{j}\left(a\left(\delta_{m}-s\right)+\varphi_{m}-\frac{\pi}{2}\right)} \mp \Delta\left(i_{m}+s\right) \\
\arg \left(G\left(i_{m}+s\right)\right)=\varphi_{m}+a\left(\delta_{m}-s\right)-\frac{\pi}{2} \mp \Delta \varphi\left(i_{m}+s\right)
\end{gathered}
$$

Fig. 10, with large values of displacements, shows the amplitude and the phase differences between the phasors of the short-range contributions (dotted lines) and the complete phasors $G\left(i_{m}\right)$ and $G\left(i_{m}+s\right)$. It should be noted that differences have opposite signs at the largest two DFT coefficients surrounding the investigated component.

As we know, the spectrum with the Hann window can be obtained by shifting and weighing summations of the rectangular window spectrum (12). Using (34) in (12), it can be written:

$$
W_{\mathrm{H}}(\theta)=\frac{\sin (\pi \theta)}{2} \cdot \mathrm{e}^{-\mathrm{j} a \theta} \cdot\left(\frac{1}{N \sin (\pi \theta / N)}-\frac{1}{2}\left(\frac{-1}{N \sin (\pi(\theta+1) / N)} \cdot \mathrm{e}^{-\mathrm{j} a}+\frac{-1}{N \sin (\pi(\theta-1) / N)} \cdot \mathrm{e}^{\mathrm{j} a}\right)\right)
$$




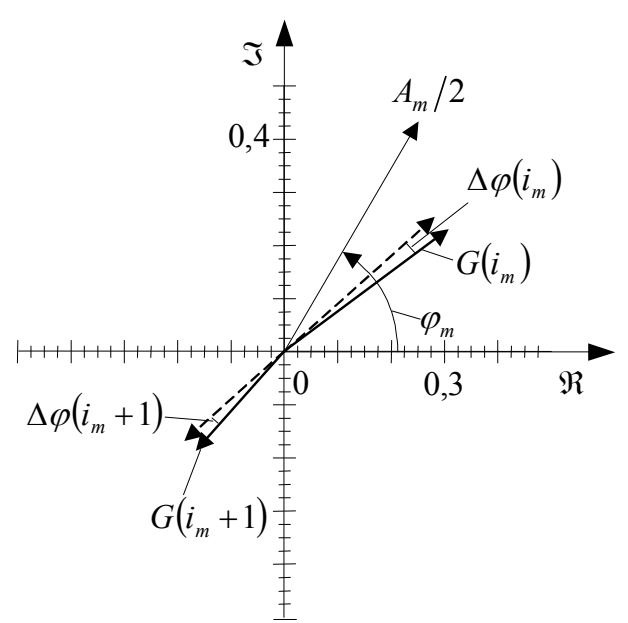

(a)

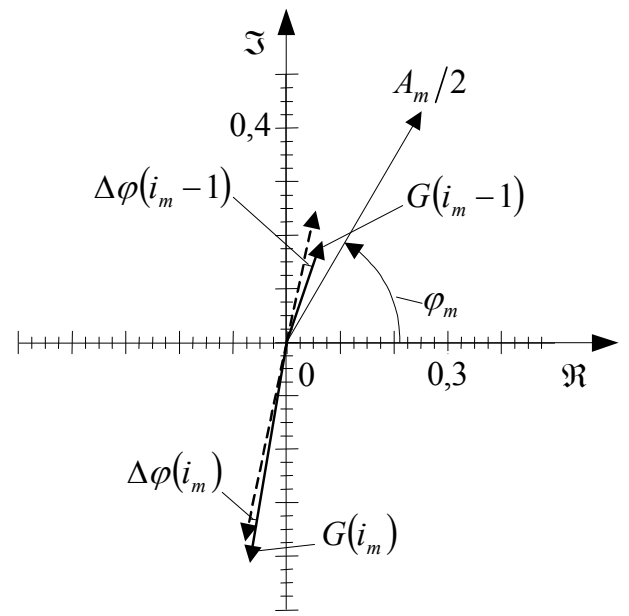

(b)

Fig. 10. Phasors diagrams for a single component $A_{m}=1, \varphi_{m}=\pi / 3$, and the rectangular window: a) $\theta_{m}=2.4, i_{m}=2, \delta_{m}=0.4$; b) $\theta_{m}=1.6, i_{m}=2, \delta_{m}=-0.4$

If we have a lot of points in the measurement set $N \gg 1$, the sine function can be approximated by $\sin (\pi \theta / N) \approx \pi \theta / N$. Considering also $\mathrm{e}^{\mathrm{j} a} \approx-1$ and $\mathrm{e}^{-\mathrm{j} a} \approx-1$, the expression in brackets can be simplified $\left(\frac{1}{\pi \theta}-\frac{1}{2}\left(\frac{1}{\pi(\theta+1)}+\frac{1}{\pi(\theta-1)}\right)\right)=\frac{1}{\pi \theta\left(1-\theta^{2}\right)}$, and we finally get:

$$
W_{\mathrm{H}}(\theta)=\frac{1}{2} \frac{\sin (\pi \theta)}{\pi \theta\left(1-\theta^{2}\right)} \cdot \mathrm{e}^{-\mathrm{j} a \theta}
$$

The largest DFT coefficient can be deduced from (5) and (40) considering $i_{m}-\theta_{m}=-\delta_{m}$ and $i_{m}+\theta_{m}=2 i_{m}+\delta_{m}$ :

$$
G_{\mathrm{H}}\left(i_{m}\right)=\frac{A_{m}}{4}\left(\frac{\sin \left(\pi \delta_{m}\right)}{\pi \delta_{m}\left(1-\delta_{m}^{2}\right)} \mathrm{e}^{\mathrm{j}\left(a\left(\delta_{m}\right)+\varphi_{m}-\frac{\pi}{2}\right)}-\frac{\sin \left(\pi \delta_{m}\right)}{\pi\left(2 i_{m}+\delta_{m}\right)\left(1-\left(2 i_{m}+\delta_{m}\right)^{2}\right)} \mathrm{e}^{-\mathrm{j}\left(a\left(2 i_{m}+\delta_{m}\right)+\varphi_{m}+\frac{\pi}{2}\right)}\right)
$$

As with the rectangular window, the second part in (41) causes additional disturbing components:

$$
\begin{gathered}
G_{\mathrm{H}}\left(i_{m}\right)=\left|G_{\mathrm{H}}\left(i_{m}\right)\right| \mathrm{e}^{\mathrm{j}\left(a\left(\delta_{m}\right)+\varphi_{m}-\frac{\pi}{2}\right)} \pm \Delta\left(i_{m}\right),\left|G_{\mathrm{H}}\left(i_{m}\right)\right|=\frac{A_{m}}{4} \frac{\sin \left(\pi \delta_{m}\right)}{\pi \delta_{m}\left(1-\delta_{m}^{2}\right)} \\
\arg \left(G_{\mathrm{H}}\left(i_{m}\right)\right)=\varphi_{i_{m}}=\varphi_{m}+a \delta_{m}-\frac{\pi}{2} \pm \Delta \varphi\left(i_{m}\right)
\end{gathered}
$$


The largest side coefficient can be expressed in short form as:

$$
\begin{gathered}
G_{\mathrm{H}}\left(i_{m}+s\right)=\left|G_{H}\left(i_{m}+s\right)\right| \mathrm{e}^{\mathrm{j}\left(a\left(\delta_{m}-s\right)+\varphi_{m}-\frac{\pi}{2}\right)} \mp \Delta\left(i_{m}+s\right) ;\left|G_{\mathrm{H}}\left(i_{m}+s\right)\right|=\frac{A_{m}}{4} \frac{\sin \left(\pi\left(s-\delta_{m}\right)\right)}{\pi\left(s-\delta_{m}\right)\left(1-\left(s-\delta_{m}\right)^{2}\right)} \\
\arg \left(G_{\mathrm{H}}\left(i_{m}+s\right)\right)=\varphi_{i_{m}+s}=\varphi_{m}+a\left(\delta_{m}-s\right)-\frac{\pi}{2} \mp \Delta \varphi\left(i_{m}+s\right)
\end{gathered}
$$

\subsubsection{Reduction of the systematic error}

In the first approximation using the rectangular window, the second term in (36) and (38) can be neglected and the phase of component can be estimated by:

$$
\begin{gathered}
\varphi_{m, \mathrm{R}}^{\mathrm{I}}=\arg \left[G\left(i_{m}\right)\right]-a \delta_{m}+\frac{\pi}{2} \\
\varphi_{m, \mathrm{R}}^{\mathrm{II}}=\arg \left[G\left(i_{m}+s\right)\right]+a\left(s-\delta_{m}\right)+\frac{\pi}{2}
\end{gathered}
$$

Another possibility is to estimate the component phase only by the phase DFT coefficient itself, where $\varphi_{m}$ is referred to the middle point of the measurement window. However, this method has the same weak point as (44) and (45), since it doesn't consider the long-range contributions of the window (Fig. 14d).

We can improve the estimation by considering the long-range contributions. Because the disturbing angle components $\Delta \varphi(*)$ in (37) and (39) are small, they can be exchanged by sine functions and approximated by quotients:

$$
\frac{\Delta \varphi\left(i_{m}\right)}{\Delta \varphi\left(i_{m}+s\right)} \cong \frac{\sin \left[\Delta \varphi\left(i_{m}\right)\right]}{\sin \left[\Delta \varphi\left(i_{m}+s\right)\right]}=\frac{\left|\Delta\left(i_{m}\right)\right|\left|G\left(i_{m}+s\right)\right|}{\left|G\left(i_{m}\right)\right|}
$$

Here, the maximal amplitude values $|*|$ from (36) and (38) are taken in approximation:

$$
\frac{\left|\Delta\left(i_{m}\right)\right|}{\left|\Delta\left(i_{m}+s\right)\right|} \cdot \frac{\left|G\left(i_{m}+s\right)\right|}{\left|G\left(i_{m}\right)\right|}=\frac{2 i_{m}+s+\delta_{m}}{2 i_{m}+\delta_{m}} \cdot \frac{\left|G\left(i_{m}+s\right)\right|}{\left|G\left(i_{m}\right)\right|}
$$

If considering only one component with the DFT coefficient index large enough $i_{m}>1$, or we want to symmetrically 'equalize' the long-range leakage contributions coming from both sides of the frequency axis, considering a multi-component signal - non-parametric approach, leakages can be equalized $\left|\Delta\left(i_{m}\right)\right| \approx\left|\Delta\left(i_{m}+s\right)\right|$ and (46) rewritten as:

$$
\frac{\Delta \varphi\left(i_{m}\right)}{\Delta \varphi\left(i_{m}+s\right)} \cong \frac{\left|G\left(i_{m}+s\right)\right|}{\left|G\left(i_{m}\right)\right|}=\frac{\delta_{m}}{s-\delta_{m}} ; \quad\left(s-\delta_{m}\right) \Delta \varphi\left(i_{m}\right) \cong \delta_{m} \Delta \varphi\left(i_{m}+s\right)
$$

The multiplication of (37) and (39) by the correction (48), and subsequent summation gives us an estimation of the phase, as an averaging of the two arguments $\varphi_{i_{m}}=\arg \left[G\left(i_{m}\right)\right]$ and $\varphi_{i_{m}+s}=\arg \left[G\left(i_{m}+s\right)\right]$ surrounding the component (Fig. 12b): 


$$
\varphi_{m, \mathrm{R}}^{\mathrm{III}}=\left(1-\left|\delta_{m}\right|\right) \cdot \varphi_{i_{m}}+\left|\delta_{m}\right| \cdot \varphi_{i_{m}+s}+\frac{\pi}{2}
$$

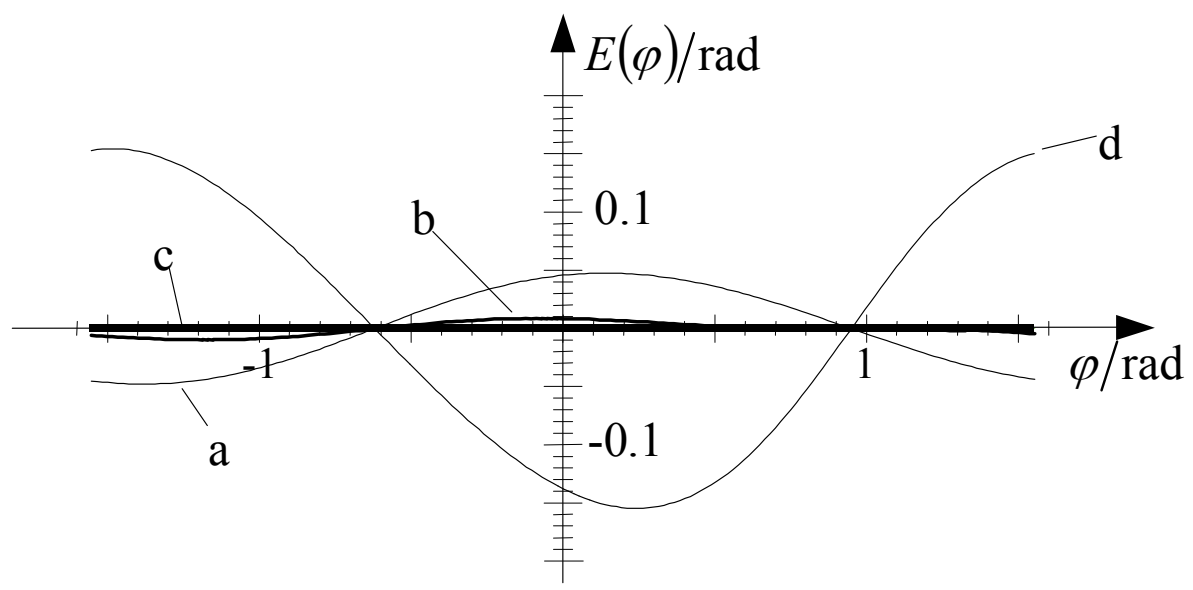

Fig. 11. Phase dependency errors at $\theta=2.2,-\pi / 2 \leq \varphi \leq \pi / 2$; Estimations: a - by (44), b - by (49), c - by (51), d - by (45)

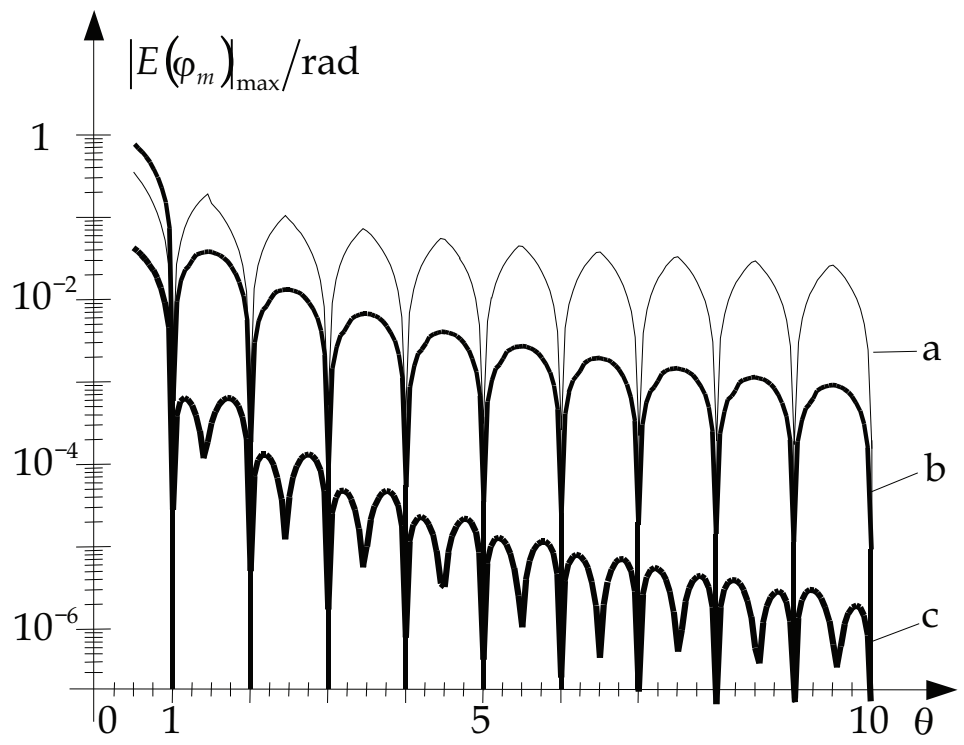

Fig. 12. Maximal systematic errors of the phase estimations with the rectangular window: a - by (44), b - by (49), c - by (51); $\theta$ is known

A better estimation can be obtained by also considering the long-range contributions in (47), when only one component is dominant in the signal - a more parametric approach (Fig. 12c): 


$$
\begin{gathered}
\frac{\Delta \varphi\left(i_{m}\right)}{\Delta \varphi\left(i_{m}+s\right)} \cong \frac{2 i_{m}+s+\delta_{m}}{2 i_{m}+\delta_{m}} \cdot \frac{\left|G\left(i_{m}+s\right)\right|}{\left|G\left(i_{m}\right)\right|}=b \cdot \frac{\left|G\left(i_{m}+s\right)\right|}{\left|G\left(i_{m}\right)\right|} \\
\varphi_{m, \mathrm{R}}^{\mathrm{IV}}=\frac{\left|G\left(i_{m}\right)\right| \varphi_{i_{m}}+b\left|G\left(i_{m}+s\right)\right| \varphi_{i_{m}+s}}{\left|G\left(i_{m}\right)\right|+b\left|G\left(i_{m}+s\right)\right|}+s a\left(\frac{b\left|G\left(i_{m}+s\right)\right|}{\left|G\left(i_{m}\right)\right|+b\left|G\left(i_{m}+s\right)\right|}-\left|\delta_{m}\right|\right)+\frac{\pi}{2}
\end{gathered}
$$

The systematic errors of the phase estimations $E=\varphi_{m}-\varphi_{0}\left(\varphi_{0}-\right.$ is the true value of the phase) are phase dependent (Fig. 11: The error curves are very close to the sine like functions). In simulations, the absolute maximum values of the errors at a given relative frequency have been searched when phase has been changed in intervals $-\pi / 2 \leq \varphi \leq \pi / 2$ (Fig. 12). The estimation errors drop with the increasing relative frequency.

Fig. 13 shows the importance of the accuracy of the frequency estimation. If the frequency is estimated by a known two-point estimation (9) the overall errors increase (Fig. 3: $\left.E_{\mathrm{c}^{*}} / E_{\mathrm{c}} \approx 200\right)$.

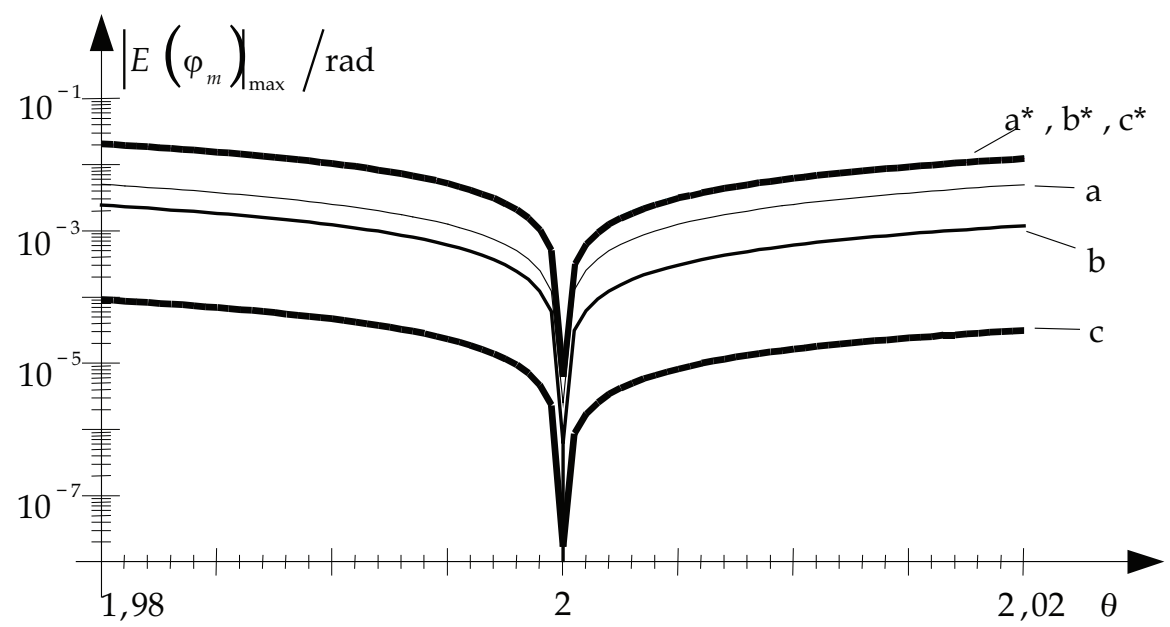

Fig. 13. Maximal systematic errors of the phase estimations with the rectangular window in the interval $1.98 \leq \theta \leq 2.02$ : a - by (44), b-by (49), c -by (51); ${ }^{*}, b^{*}, c^{*}-\theta$ is estimated by (9)

Using the Hann window, the expressions for phase have the same forms as for the rectangular window when the second term in (41) and (43) is neglected:

$$
\begin{gathered}
\varphi_{m, \mathrm{H}}^{\mathrm{I}}=\varphi_{i_{m}}-a \delta_{m}+\frac{\pi}{2} \\
\varphi_{m, \mathrm{H}}^{\mathrm{II}}=\varphi_{i_{m}+s}+a\left(s-\delta_{m}\right)+\frac{\pi}{2}
\end{gathered}
$$

We can again improve the estimation by considering the long-range contributions, which have the following properties: 


$$
\begin{gathered}
\frac{\Delta \varphi\left(i_{m}\right)}{\Delta \varphi\left(i_{m}+s\right)} \cong \frac{\sin \left(\Delta \varphi\left(i_{m}\right)\right)}{\sin \left(\Delta \varphi\left(i_{m}+s\right)\right)}=\frac{\left|\Delta\left(i_{m}\right)\right|}{\left|G_{\mathrm{H}}\left(i_{m}\right)\right|} \frac{\left|G_{\mathrm{H}}\left(i_{m}+s\right)\right|}{\left|\Delta\left(i_{m}+s\right)\right|}= \\
=\frac{\left(2 i_{m}+s+\delta_{m}\right)\left(1-\left(2 i_{m}+s+\delta_{m}\right)^{2}\right)}{\left(2 i_{m}+\delta_{m}\right)\left(1-\left(2 i_{m}+\delta_{m}\right)^{2}\right)} \cdot \frac{\left|G_{\mathrm{H}}\left(i_{m}+s\right)\right|}{\left|G_{\mathrm{H}}\left(i_{m}\right)\right|}
\end{gathered}
$$

In the non-parametric approach, one can also equalize $\left|\Delta\left(i_{m}\right)\right| \approx\left|\Delta\left(i_{m}+s\right)\right|$ and (54) can be rewritten as:

$$
\frac{\Delta \varphi\left(i_{m}\right)}{\Delta \varphi\left(i_{m}+s\right)} \cong \frac{\left|G_{\mathrm{H}}\left(i_{m}+s\right)\right|}{\left|G_{\mathrm{H}}\left(i_{m}\right)\right|}=\frac{\delta_{m}\left(1-\delta_{m}^{2}\right)}{\left(s-\delta_{m}\right)\left(1-\left(s-\delta_{m}\right)^{2}\right)}=\frac{1+s \delta_{m}}{2-s \delta_{m}}
$$

If we equalize $\left(2-s \delta_{m}\right) \Delta \varphi\left(i_{m}\right) \cong\left(1+s \delta_{m}\right) \Delta \varphi\left(i_{m}+s\right)$, the equations (42) and (43) can be multiplied by corrections and added:

$$
\begin{gathered}
\varphi_{m, \mathrm{H}}^{\mathrm{I}}=\varphi_{i_{m}}-a \delta_{m}+\frac{\pi}{2} \pm \Delta \varphi\left(i_{m}\right) \quad \mid \cdot\left(2-s \delta_{m}\right) \\
\varphi_{m, \mathrm{H}}^{\mathrm{II}}=\varphi_{i_{m}+s}+a\left(s-\delta_{m}\right)+\frac{\pi}{2} \mp \Delta \varphi\left(i_{m}+s\right) \mid \cdot\left(1+s \delta_{m}\right) \\
\varphi_{m, \mathrm{H}}^{\mathrm{III}}=\frac{\left(2-s \delta_{m}\right) \varphi_{i_{m}}+\left(1+s \delta_{m}\right) \varphi_{i_{m}+s}}{3}+\frac{a}{3}\left(s-2 \delta_{m}\right)+\frac{\pi}{2}
\end{gathered}
$$

When the sign of the displacement is positive $s=1$, the phase estimation (56) is made with arguments $\varphi_{i_{m}}$ and $\varphi_{i_{m}+1}$ :

$$
\varphi_{m, \mathrm{H}}^{\mathrm{III}}\left(\varphi_{i_{m}}, \varphi_{i_{m}+1}\right)=\frac{\left(2-\delta_{m}\right) \varphi_{i_{m}}+\left(1+\delta_{m}\right) \varphi_{i_{m}+1}}{3}+\frac{a}{3}\left(1-2 \delta_{m}\right)+\frac{\pi}{2},
$$

and when the sign is negative $s=-1$, the estimation is done with $\varphi_{i_{m}}$ and $\varphi_{i_{m}-1}$ :

$$
\varphi_{m, \mathrm{H}}^{\mathrm{III}}\left(\varphi_{i_{m}}, \varphi_{i_{m}-1}\right)=\frac{\left(2+\delta_{m}\right) \varphi_{i_{m}}+\left(1-\delta_{m}\right) \varphi_{i_{m}-1}}{3}-\frac{a}{3}\left(1+2 \delta_{m}\right)+\frac{\pi}{2}
$$

The phase estimation can be improved further with averaging of the estimations by (57) and (58). With this averaging we get the three-point estimation (Fig. 14c):

$$
\begin{gathered}
\varphi_{m, \mathrm{H}}^{\mathrm{IV}}\left(\varphi_{i_{m}}, \varphi_{i_{m}+1}, \varphi_{i_{m}-1}\right)=\frac{\varphi_{m, \mathrm{H}}^{\mathrm{III}}\left(\phi_{i_{m}}, \varphi_{i_{m}+1}\right)+\varphi_{m, \mathrm{H}}^{\mathrm{III}}\left(\varphi_{i_{m}}, \varphi_{i_{m}-1}\right)}{2} \\
\varphi_{m, \mathrm{H}}^{\mathrm{IV}}=\frac{\left(1-\delta_{m}\right) \varphi_{i_{m}-1}+4 \varphi_{i_{m}}+\left(1+\delta_{m}\right) \varphi_{i_{m}+1}}{6}-\frac{2 a \delta_{m}}{3}+\frac{\pi}{2}
\end{gathered}
$$


The best results are obtained from the three-point estimation using the Hann window, when the frequency is known (Fig. 14c: $E(\varphi)_{\max } \leq 1.7 \cdot 10^{-5} \mathrm{rad} \approx 1 \mathrm{~m}^{\circ} \Leftarrow \theta>5.5$ ). If the frequency has to be estimated, the overall error increases, but it is still under the error level of the one-point estimation (Fig. 14: $\mathrm{b}^{*}<\mathrm{a} \Leftarrow \theta>5.5$ ). In Fig. 14, for error curve $\mathrm{b}^{*}$, the frequency is estimated by the three-point interpolation (11).

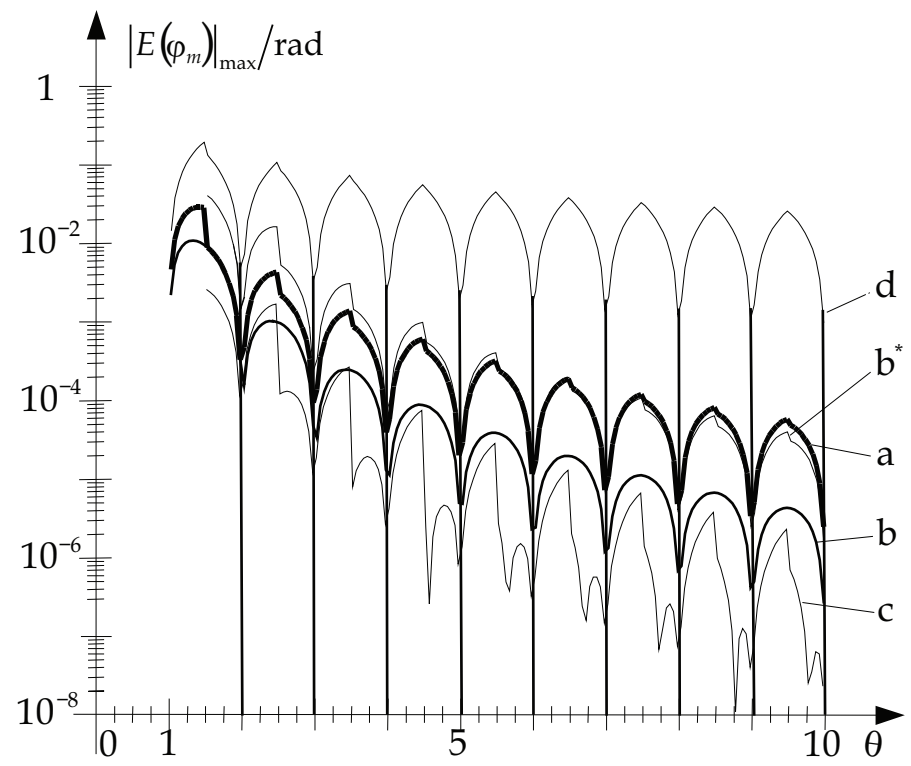

Fig. 14. Maximal systematic errors of the phase estimations with Hann window: a - onepoint by (52), $\mathrm{b}$ - two-point by (56), c - three-point by (60), $\theta$ is known; $\mathrm{b}^{*}-\theta$ is estimated by (11); and estimations using only phase DFT coefficient: $d$ - the rectangular window, $\mathrm{a}-$ the Hann window

\subsubsection{Uncertainty of the phase estimation}

The uncertainty propagation through the DFT procedure is well known $\sigma_{R}=\sigma_{I}=\sigma_{|G(i)|}=\sigma_{\mathrm{DFT}}=\sigma_{t} /(N \sqrt{2}) \sqrt{\sum_{k=0}^{N-1} w^{2}(k)}$ (Agrež, 2007), where we use $R(i)=\operatorname{Re}[G(i)]$ and $I(i)=\operatorname{Im}[G(i)]$ for the real and imaginary parts of the DFT, respectively, and $|G(i)|=\sqrt{R^{2}(i)+I^{2}(i)}$ for the amplitude, and $\varphi(i)=\arg [G(i)]=\tan ^{-1}(I(i) / R(i))$ for the phase. The phase uncertainty is equal to the uncertainty of the DFT procedure scaled by the amplitude coefficient $\sigma_{\varphi(i)}=\sigma_{\mathrm{DFT}} /|G(i)|$ :

$$
c_{R}(i)=\frac{\partial \varphi(i)}{\partial R(i)}=-\frac{I(i)}{|G(i)|^{2}} ; \quad c_{I}(i)=\frac{\partial \varphi(i)}{\partial I(i)}=\frac{R(i)}{|G(i)|^{2}}
$$




$$
\sigma_{\varphi(i)}^{2}=\left(c_{R} \sigma_{R}\right)^{2}+\left(c_{I} \sigma_{I}\right)^{2}=\sigma_{\mathrm{DFT}}^{2} \frac{I^{2}+R^{2}}{|G(i)|^{4}}=\frac{\sigma_{\mathrm{DFT}}^{2}}{|G(i)|^{2}}
$$

It is evident that the standard uncertainty of the phase depends on the amplitude of the component. Moreover, in non-coherent sampling it changes with displacement $\delta$ as we see in the following examples.

First, we consider that frequency is known $\left(\sigma_{\delta}=0\right)$. For ease of understanding, one can omit index $m$ in (44) $\varphi_{\mathrm{a}}=\varphi(i)-a \cdot \delta+\pi / 2$, and from (62) we get (Fig. 15a):

$$
\frac{\sigma_{\varphi_{\mathrm{a}}}}{\sigma_{\mathrm{DFT}}}=\frac{1}{|G(i)|}
$$

For the second estimation by $\varphi_{\mathrm{b}}=(1-s \delta) \varphi(\mathrm{i})+s \delta \varphi(\mathrm{i}+\mathrm{s})+\pi / 2$ (49) one needs sensitivity coefficients associated with the real and imaginary coefficients for two spectral lines $i$, $i+s$ :

$$
\begin{gathered}
c_{R_{\mathrm{b}}, i}=\frac{\partial \varphi_{\mathrm{b}}(i)}{\partial R(i)}=(1-s \delta) c_{R}(i) ; \quad c_{I_{\mathrm{b}}, i}=\frac{\partial \varphi_{\mathrm{b}}(i)}{\partial I(i)}=(1-s \delta) c_{I}(i) \\
c_{R_{\mathrm{b}}, i+s}=\frac{\partial \varphi_{\mathrm{b}}(i+s)}{\partial R(i+s)}=s \delta c_{R}(i+s) ; \quad c_{I_{\mathrm{b}}, i+s}=\frac{\partial \varphi_{\mathrm{b}}(i+s)}{\partial I(i+s)}=s \delta c_{I}(i+s)
\end{gathered}
$$

As the correlation coefficients for the rectangular window $r(R(i), R(i+s))$ and $r(I(i), I(i+s))$ are zero, and standard uncertainties are equal $\sigma_{R, *}=\sigma_{I, *}=\sigma_{\mathrm{DFT}}(*=i, i+s)$ we can write according to (JCGM, 2008) (Fig. 15b):

$$
\left(\frac{\sigma_{\varphi_{\mathrm{b}}}}{\sigma_{\mathrm{DFT}}}\right)^{2}=c_{R_{\mathrm{b}}, i}^{2}+c_{I_{\mathrm{b}}, i}^{2}+c_{R_{\mathrm{b}}, i+s}^{2}+c_{I_{\mathrm{b}}, i+s}^{2} ; \quad \frac{\sigma_{\varphi_{\mathrm{b}}}}{\sigma_{\mathrm{DFT}}}=\sqrt{((1-s \delta) /|G(i)|)^{2}+(\delta /|G(i+s)|)^{2}}
$$

In calculations of the four sensitivity coefficients for the third estimation (51), one needs partial sensitivity coefficients for the amplitude $\partial|G(*)| / \partial R(*)=R(*) /|G(*)|$, $\partial|G(*)| / \partial I(*)=I(*) /|G(*)|$ and for the phase coefficients $\partial \varphi(*) / \partial R(*), \partial \varphi(*) / \partial I(*)(61)$, since all of them contribute in the estimation:

$$
\begin{gathered}
\varphi_{\mathrm{c}}=\frac{|G(i)| \varphi(\mathrm{i})+b|G(i+s)| \varphi(\mathrm{i}+\mathrm{s})}{|G(i)|+b|G(i+s)|}+s a\left(\frac{b|G(i+s)|}{|G(i)|+b|G(i+s)|}-s \delta\right)+\frac{\pi}{2} \\
c_{R_{\mathrm{c}}, *}=\frac{\partial \varphi_{\mathrm{c}}(*)}{\partial R(*)} ; \quad c_{I_{\mathrm{c}}, *}=\frac{\partial \varphi_{\mathrm{c}}(*)}{\partial I(*)}
\end{gathered}
$$

In this case the uncertainty is close to the uncertainty of the estimation by (49) (Fig. 15c). 
The price for the effective leakage reduction is in the increase of the estimation uncertainties related to the unbiased CRB. Ratios of the uncertainties of the phase estimations related to the CRB (68), are between 1 and 1.7 symmetrically, depending upon the term $\delta$ at higher values of the relative frequency $\theta$ (Fig. 15).

$$
\sigma_{\varphi_{m}} \geq 2 \frac{1}{\sqrt{S N R}} \frac{1}{\sqrt{N}}=\sigma_{\mathrm{CRB}, \varphi}
$$

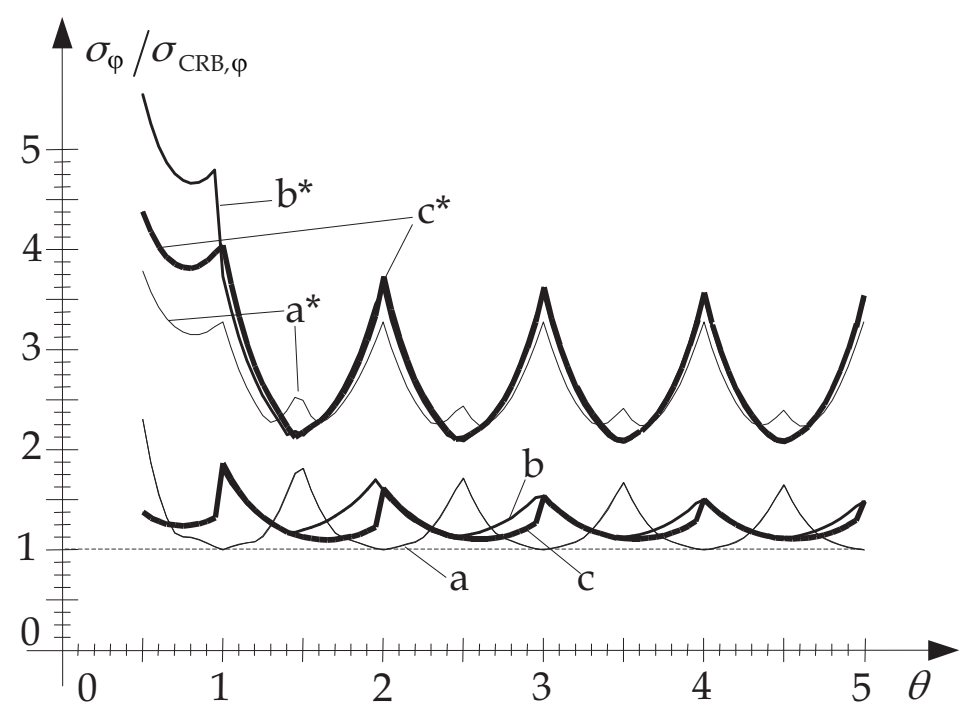

Fig. 15. Ratios of the uncertainties of the phase estimations with rectangular window related to the CRB (68). Estimations: a - by (44), b - by (49), c - by (51), $\theta$ is known; $\mathrm{a}^{*}, \mathrm{~b}^{*}, \mathrm{c}^{*}-\theta$ is estimated by $(9 b)$

The uncertainties of the estimations increase if one needs to also estimate the displacement term $\delta(|G(i)|,|G(i+s)|)$. In estimation algorithms (44), (49), and (51), one also needs partial sensitivity coefficients for the displacement terms $\partial \delta / \partial|G(i)|$ and $\partial \delta / \partial|G(i+s)|$. Fig. 15 shows that uncertainties of the estimations increase for a factor $2 \div 2.3$ if frequency is estimated by $(9 \mathrm{~b})$. The uncertainties of the estimations where frequency has to be estimated first, are very close to each other (Fig. 15: $E_{\mathrm{a}^{*}} \approx E_{\mathrm{b}^{*}} \approx E_{\mathrm{c}^{*}}$ ) at higher values of $\theta$. For the Hann window, the uncertainty levels increase (62), as the main amplitude coefficient decreases by a factor of 2 with respect to the rectangular window.

\section{Conclusion}

In chapter, the non-parametric interpolated DFT algorithms for emulating coherent sampling are described. The advantages of the DFT interpolations for the frequency, amplitude, and phase of the signal component are identified. Interpolations where the longrange leakage is considered, illustrate a decrease in systematic effects. The algorithms retain all benefits of the DFT approach and improve the estimation accuracy adaptively for a 
particular component as a function of its frequency position. The weights emphasize the DFT coefficients of the spectrum peak related to the investigated component. The spectrum of the window used must be formally well-known, like the Hann window, for a better analytical expression. Interpolation with a larger number of the DFT coefficients decreases the systematic errors. It can be concluded, that if we selectively use a different number of the DFT coefficients in the interpolation algorithms for a particular component of the signal, we adapt the apparent window shape for that component. A trade-off between a reduction in systematic error of the parameter estimation and the uncertainty of the estimated results is highlighted. The use of a suitable interpolation algorithm depends on the level of the noise floor of the acquisition channel, or better $S N R$, and on the position of the frequency component along the frequency axis.

\section{References}

Agrež D. (2002). Weighted Multi-Point Interpolated DFT to Improve Amplitude Estimation of Multi-Frequency Signal. IEEE Transactions on Instrumentation and Measurement, Vol. 51, No. 2, April 2002, pp. 287-292, ISSN 0018-9456

Agrež, D. (2007). Dynamics of frequency estimation in the frequency domain. IEEE Transactions on Instrumentation and Measurement, Vol. 56, No. 6, December 2007, pp. 2111-2118, ISSN 0018-9456

Belega, D. \& Dallet, D. (2009). Multifrequency signal analysis by interpolated DFT method with maximum sidelobe decay windows. Measurement, Vol. 42, No. 3, 2009, pp. 420426, April 2009, ISSN 0263-2241

D’Antona G. \& Ferrero, A. (2006). Digital Signal Processing for Measurement Systems. Theory and Applications, Springer Science 2006, ISBN-10: 0-387-24966-4

Gabor, D. (1946). Theory of communication. Journal of the IEEE, Vol. 93, pp. 429-457

Harris, F. J. (1978). On the use of windows for harmonic analysis with the discrete Fourier transform. Proceedings of the IEEE, Vol. 66, No. 1, January 1978, pp. 51-83, ISSN 0018-9219

JCGM (2008). Evaluation of measurement data - Guide to the expression of uncertainty in measurement. Joint Committee for Guides in Metrology [JCGM] 100:2008, First edition, September 2008

Novotný, M. \& Sedláček, M. (2010). The influence of window sidelobes on DFT-based multifrequency signal measurement. Computer Standards \& Interfaces, Vol. 32, No. 3, March 2010, pp. 110-118, ISSN 0920-5489

Petri, D. (2002). Frequency-domain testing of waveform digitizers. IEEE Transactions on Instrumentation and Measurement, Vol. 51, No. 3, 2002, pp. 445-453, ISSN 00189456

Schoukens, J., Pintelon, R. \& Van hamme, H. (1992). The interpolated fast Fourier transform: A comparative study. IEEE Transactions on Instrumentation and Measurement, Vol. 41, No. 2, April 1992, pp. 226-232, ISSN 0018-9456

Siebert, W. McC. (1986) Circuits, Signals and Systems, The MIT Press, ISBN 0-262-19229-2, McGraw-Hill, Cambridge, New York, ..., pp. 497-502 
Solomon, O. M. (1992). The effects of windowing and quantization error on the amplitude of frequency-domain functions. IEEE Transactions on Instrumentation and Measurement, Vol. 41, No. 6, December 1992, pp. 932-937, ISSN 0018-9456

Widrow, B. \& Kollar, I. (2008) Quantization Noise, Cambridge Univ. Press 2008, ISBN 978-0521-88671-0, Cambridge, New York, 


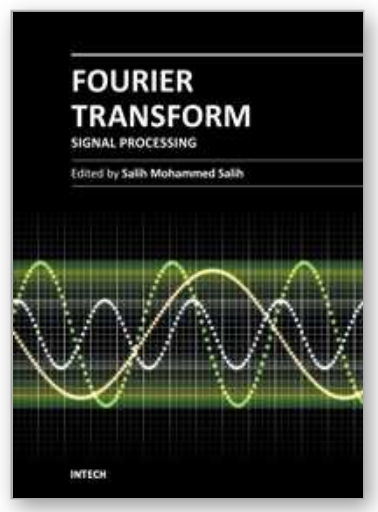

\author{
Fourier Transform - Signal Processing \\ Edited by Dr Salih Salih
}

ISBN 978-953-51-0453-7

Hard cover, 354 pages

Publisher InTech

Published online 11, April, 2012

Published in print edition April, 2012

The field of signal processing has seen explosive growth during the past decades; almost all textbooks on signal processing have a section devoted to the Fourier transform theory. For this reason, this book focuses on the Fourier transform applications in signal processing techniques. The book chapters are related to DFT, FFT, OFDM, estimation techniques and the image processing techqniques. It is hoped that this book will provide the background, references and the incentive to encourage further research and results in this area as well as provide tools for practical applications. It provides an applications-oriented to signal processing written primarily for electrical engineers, communication engineers, signal processing engineers, mathematicians and graduate students will also find it useful as a reference for their research activities.

\title{
How to reference
}

In order to correctly reference this scholarly work, feel free to copy and paste the following:

Dusan Agrez (2012). Non-Parametric Estimation of the Periodic Signal Parameters in the Frequency Domain, Fourier Transform - Signal Processing, Dr Salih Salih (Ed.), ISBN: 978-953-51-0453-7, InTech, Available from: http://www.intechopen.com/books/fourier-transform-signal-processing/non-parametric-estimation-of-theperiodic-signal-parameters-in-the-frequency-domain

\section{INTECH}

open science | open minds

\section{InTech Europe}

University Campus STeP Ri

Slavka Krautzeka 83/A

51000 Rijeka, Croatia

Phone: +385 (51) 770447

Fax: +385 (51) 686166

www.intechopen.com

\section{InTech China}

Unit 405, Office Block, Hotel Equatorial Shanghai

No.65, Yan An Road (West), Shanghai, 200040, China

中国上海市延安西路65号上海国际贵都大饭店办公楼 405 单元

Phone: +86-21-62489820

Fax: +86-21-62489821 
(C) 2012 The Author(s). Licensee IntechOpen. This is an open access article distributed under the terms of the Creative Commons Attribution 3.0 License, which permits unrestricted use, distribution, and reproduction in any medium, provided the original work is properly cited. 